\title{
Genomic analysis of NAC transcription factors in banana (Musa acuminata) and definition of NAC orthologous groups for monocots and dicots
}

\author{
Albero Cenci • Valentin Guignon • \\ Nicolas Roux $\cdot$ Mathieu Rouard
}

Received: 12 July 2013 / Accepted: 24 December 2013 / Published online: 26 February 2014

(c) The Author(s) 2014. This article is published with open access at Springerlink.com

\begin{abstract}
Identifying the molecular mechanisms underlying tolerance to abiotic stresses is important in crop breeding. A comprehensive understanding of the gene families associated with drought tolerance is therefore highly relevant. NAC transcription factors form a large plant-specific gene family involved in the regulation of tissue development and responses to biotic and abiotic stresses. The main goal of this study was to set up a framework of orthologous groups determined by an expert sequence comparison of NAC genes from both monocots and dicots. In order to clarify the orthologous relationships among NAC genes of different species, we performed an in-depth comparative study of four divergent taxa, in dicots and monocots, whose genomes have already been completely sequenced: Arabidopsis thaliana, Vitis vinifera, Musa acuminata and Oryza sativa. Due to independent evolution, NAC copy number is highly variable in these plant genomes. Based on an expert NAC sequence comparison, we propose forty orthologous groups of NAC sequences that were probably derived from an ancestor gene present in the most recent common ancestor of dicots and monocots. These orthologous groups provide a curated resource for large-scale protein sequence annotation of NAC transcription factors. The established orthology relationships also provide a useful reference for NAC function studies in newly sequenced genomes such as M. acuminata and other plant species.
\end{abstract}

Electronic supplementary material The online version of this article (doi:10.1007/s11103-013-0169-2) contains supplementary material, which is available to authorized users.

A. Cenci $(\bowtie) \cdot$ V. Guignon $\cdot$ N. Roux $\cdot$ M. Rouard

Bioversity International, Commodity Systems and Genetic

Resources Programme, Parc Scientifique Agropolis II,

1990 Boulevard de la Lironde, 34397 Montpellier Cedex 5,

France

e-mail: a.cenci@cgiar.org
Keywords Comparative genomics - NAC transcription factors $\cdot$ Phylogenetic analysis · Gene family · Expert annotation $\cdot$ Gene duplication

\section{Introduction}

Identifying the molecular mechanisms underlying tolerance to abiotic stresses is important in crop breeding. A comprehensive understanding of the gene families associated with drought tolerance is therefore highly relevant. The NAC gene family is one of the largest groups of plant transcription factors (TFs), which is known to regulate biotic and abiotic stress-responses such as osmotic stress and various plant developmental processes. NAC proteins are plant-specific TFs, and the NAC family has been recently reviewed by Puranik et al. (2012). NAC genes were originally characterized in a petunia NAM mutant (Souer et al. 1996) and then in Arabidopsis CUC (Aida et al. 1997) and ATAF mutants (GenBank accession numbers X74755 and $\underline{X 74756})$. Two parts can be distinguished in the structure of NAC proteins: the NAC domain (InterPro IPR003441), in the N-terminal region, subdivided in five well-conserved subdomains (A-E); and the transcription regulatory regions (TRRs), in the C-terminal region, which is very variable in sequence and in length. The NAC domain is involved in dimerization and DNA binding, whereas the TRR region plays the role of transcription activator or repressor (Puranik et al. 2012). Evolutionary studies have been done on NAC genes for all major groups of land plants, and it has been shown that some NAC subfamilies were already present in early diverged land plants (Zhu et al. 2012).

Whole genome analyses of the NAC gene family have been performed in several species (Arabidopsis thaliana, Oryza sativa, Vitis vinifera, Populus trichocarpa, Glycine 
soja, Setaria italica) (Ooka and Satoh 2003; Fang et al. 2008; Nuruzzaman et al. 2010; Wang et al. 2013; Hu et al. 2010; Le et al. 2011; Puranik et al. 2013), as well as in a subset of viridiplantae species (Zhu et al. 2012). The number of NAC members at the genome level varies with the studied species, from 30 , in the early divergent land plants, to more than 160 in P. trichocarpa. NAC genes have been classified using phylogenetic analyses in a variable number of groups and subgroups according to the species. The comparison of NAC sequences of $A$. thaliana and $O$. sativa have shown that some NAC members in these two species derived from common ancestors that existed before monocots and dicots diverged (Nuruzzaman et al. 2010).

Whole genome duplications (WGDs) are an important evolutionary feature of plant genomes. Most plant taxa have experienced at least one WGD during their evolution (Van de Peer et al. 2009). A consequence of a WGD is the doubling of all genes. After a WGD event, genomes start to lose (by deletion or pseudogenization) the redundant copies of most of their genes, in a long evolutionary process (fractionation), but some duplicated copies are retained and fixed with modified functional properties. Moreover, differences in gene retention according with their function have been reported (Blanc and Wolfe 2004; Maere 2005). Consequently, in a multigenic family, the number of members in species that experienced independent WGD events can be highly variable. This variability is correlated with the number of WGDs that their genomes experienced in their evolution, the time elapsed from these events, the evolution rate and other evolutionary factors specific to each taxon.

The main goal of this study was to set up a framework of Orthologous Groups (OGs) determined by an expert sequence comparison of NAC genes from both monocots (O. sativa and Musa acuminata) and dicots ( $V$. vinifera and $A$. thaliana). A. thaliana and $V$. vinifera were chosen as dicot representatives because the former is a model plant species, while the genome of the latter contains a low number of NAC genes and appears to evolve more slowly than genomes of other dicot taxa (Cenci et al. 2010; 2013; Yue et al. 2010). O. sativa, belonging to the Poales clade, was selected as a monocot model species. Instead of another gramineae, we chose $M$. acuminata, a member of the Zingiberales clade of monocots which diverged early from the Poales clade. Moreover, the sequence of the $M$. acuminata DH Pahang genome was recently published and its analyses uncovered one ancient and two more recent WGDs (D'Hont et al. 2012). In banana, six members of the NAC family involved in fruit ripening have been comprehensively characterized (Shan et al. 2012). Among the species considered in the present study, $O$. sativa and $M$. acuminata experienced three independent WGDs in the evolution of their genome (D'Hont et al. 2012). V. vinifera and A. thaliana underwent a genome triplication ( $\mathrm{\gamma}$ WGD, common to all the core eudicots) (Jaillon et al. 2007) and the A. thaliana genome experienced two additional and more recent WGDs (Van de Peer et al. 2009). WGDs and gene retention lead to multigenic gene families in which OG detection using automatic approaches is challenging. The members belonging to each OG that was defined in this process are supposed to derive from the same ancestor gene existing before the divergence of monocots and dicots, and possibly to share the same function. Our classification intends to be a reference for all monocot and dicot species, to facilitate the identification of the most interesting NAC genes in species for which functional information is lacking.

\section{Methods}

\section{Sequence retrieval}

The sequences of NAC protein-coding genes were extracted from gene family database GreenPhylDB (Rouard et al. 2011) based on the presence of the NAM (No apical meristem) InterPro domain (IPR003441). Excluding splice forms, 82, 111, 146 and 172 sequences were selected for V. vinifera, A. thaliana, O. sativa, and M. acuminata, respectively.

Datasets from $V$. vinifera, A. thaliana, and $O$. sativa were compared with sequences analysed in previous studies. No additional NAC members were found for $V$. vinifera [74 members (Wang et al. 2013)], although only 69 sequences were considered in this study. Five sequences were excluded from the analyses: VvNAC35 and VvNAC38 because they are not NAC but VOZ transcription factors; VvNAC72 because it was identified as a cellulose synthaselike protein G3; and VvNAC50 and VvNAC66 because they are likely pseudogenes.

Among the $105 \mathrm{~A}$. thaliana NAC members analysed by Ooka and Satoh (2003), splice forms of five genes were eliminated (ANAC021/022, 034/035, 038/039, 050/051, and 079/080). Among the GreenPhylDB-extracted sequences, after the eliminating the NAC pseudogenes, three sequences (At3g12910.1, At3g12977.1, At4g35580.2) which were not included in the study of Ooka and Satoh (2003) were added to the A. thaliana NAC members in this study. Comparisons between $O$. sativa NAC sequences extracted from GreenPhylDB with the 138 and the 151 members found by Fang et al. (2008) and Nuruzzaman et al. (2010), respectively, provided 165 independent sequences for our study. The previously reported $M$. acuminata NAC sequences had all been scanned for the presence of the conserved NAC domain using interproscan searches (http://www.ebi.ac.uk/ Tools/pfa/iprscan/). The sequences used in the present study are provided in Online Resources 1. 
Fig. 1 Inference of the orthologous groups with similaritybased approach. Empty and filled shapes indicate orthologous copies in two different species. a Schematic representation of the amplification of a multi-copy family in two species. Arrows connect copies of an OG in a species with the less divergent copy of the other species. b Expected Blastp results for an OG. Arrows connect copies of a species with the best Blastp hit in the other species

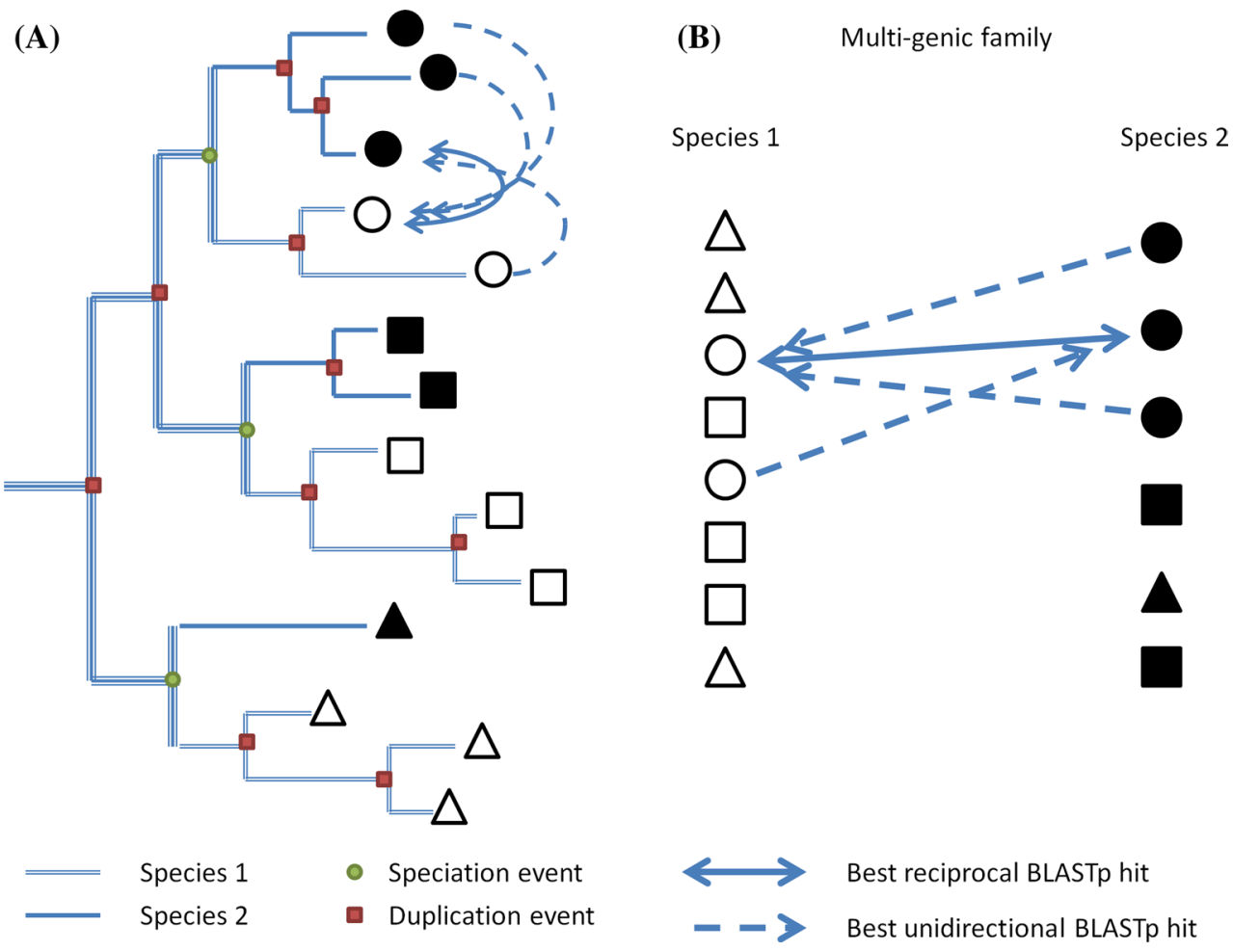

\section{Expert annotation of NAC sequences}

Since the $M$. acuminata NAC sequences were the result of an automatic annotation, an expert revision of the gene structure of this family was conducted. In order to detect additional InterPro protein domain IPR003441 not included in the annotated genes, a tBlastn search was performed on the $M$. acuminata pseudo-chromosome sequences with a sample of NAC genes. Banana sequences were curated using the Banana Genome Hub (Droc et al. 2013) and more specifically with the Artemis software (Carver et al. 2008) connected to the Community Annotation System (Guignon et al. 2012). Modifications of the gene structures were based on transcripts, when available, and protein similarity with published NAC genes of other plant species. The revised annotations are publically available via the Banana Genome Hub. Some modifications of published NAC annotations for A. thaliana, $V$. vinifera and $O$. sativa were also performed. The modified sequences are reported in Online Resources 1 and a concise description of modifications is provided in Online Resources 2.

\section{Analysis of NAC duplication in Musa genome}

NAC genes separated by not more than 10 other genes were considered as tandem duplications. NACs generated by segmental duplication of the Musa genome were determined by the analysis of the output file (Online Resources 3) of the CoGe SynMap program (http://genomevolution.
org/CoGe/SynMap.pl) obtained using a default parameter (Lyons et al. 2008).

\section{Expert inference of the OGs}

Orthologous group (OG) reconstruction was based on sequence similarity inferred from protein-protein BLAST analyses (analyses performed with default Blastp parameters) that were all carefully examined by human expertise (Fig. 1). The method used is based on two assumptions. Firstly, the best Blastp hit indicates the lowest protein distance from the query sequence. Secondly, for each member of the query species, the smallest protein distance will be found with an orthologous gene of the subject species, although, this second assumption cannot be true in all circumstances, particularly for rapid sequence evolution of the orthologous gene(s). However, since all the NAC genes have a similar function (regulation of gene transcription), dramatic differences in their evolution rates are expected to be infrequent.

The orthologous inference was performed in two steps. First, all the NAC amino acid sequences of A. thaliana, $M$. acuminata and $O$. sativa were grouped with the $V$. vinifera NAC member having the higher Blastp score. Then, Blastp analyses were performed for all the other "species by species" combinations to verify the highest sequence-similarity among the sequences of each group. When inconsistencies were found (i.e. the best NAC protein found for the three species belonged to two different groups) the majority 
rule was applied and the query sequence included inside the group containing the two best hits. The Blastp analyses of $V$. vinifera NAC sequences also allowed grouping those genes never found having best Blastp hits with the other species. Sequences having low Blastp scores with other species (see results) were considered species-specific and not considered in subsequent comparative analyses.

\section{OrthoMCL clustering}

The whole set of NAC sequences included in the OGs were clustered using OrthoMCL (Li et al. 2003) after Blastp all vs all (e-value $1 \mathrm{e}-10)$. The inflation parameter was gradually increased from 1.5 (default) to 14 , to vary levels of granularity and until we reached the number of OGs identified by the expert annotation. Then, the resulting groups were compared to check if the manually obtained results were reproducible by a common strategy for orthology group detection (i.e. OrthoMCL).

\section{Phylogenetic analyses}

Phylogenetic analyses were performed using an in-house phylogenetic workflow powered by Galaxy (available at http://gohelle.cirad.fr/galaxy/u/reviewer/w/greenphyl-phylogenomic-analysis-workflow) that reproduces the main steps of the GreenPhyl pipeline. These steps included: multiple alignment (MAFFT v.6 (Katoh and Toh 2008)); masking (GBLOCKS (Talavera and Castresana 2007)). Gene tree reconstruction was performed using PhyML (Guindon and Gascuel 2003) with tree improvement with best of NNI and SPR methods with aLRT support (Anisimova and Gascuel 2006) steps. In order to optimize the resolution of the global phylogeny, we used ProtTest v3 (Darriba et al. 2011) to define the best substitution model. Since the JTT model resulted slightly better than LG, it was chosen for the phylogeny analysis of the whole set of NAC sequences. The gene trees were reconciled using RAPGreen (Dufayard et al. 2005) using a species tree that is compliant with NCBI taxonomy (Online resources 4). All the multiple alignments and gene trees were deposited in Treebase (http://purl.org/ phylo/treebase/phylows/study/TB2:S14688)

\section{Results}

Number of NAC genes in M. acuminata genome

Among the 36,542 automatically predicted genes in the M. acuminata genome, 172 contained the InterPro protein domain IPR003441. To improve their structural annotation, all these sequences were manually curated, as necessary. Additional genomic regions containing the domain
IPR003441 were also detected and analyzed. Globally, our analysis resulted in 167 potentially functional NAC genes (Online Resources 1) that we retained for this study. Five pseudogenes and a number of remnants were also found in the $M$. acuminata genome. We then refined the selection of NAC sequences for other species, taking into account previous publications dedicated to NAC transcription factors (see "Methods").

\section{NAC member duplication in M. acuminata}

The banana genome contains the largest number of NAC genes among the already sequenced genomes of angiosperms. Despite the large number of NAC genes observed, only twelve tandem duplication regions (involving 27 genes) were detected in M. acuminata. By contrast, at least 18 segmental duplications (involving 43 NAC genes) were detected by CoGe SynMap (Online Resources 3), that originated from one of the last two WGDs that occurred for the Musa genome (D'Hont et al. 2012).

\section{Expert orthologous grouping of NAC sequences}

The $V$. vinifera $\mathrm{NAC}$ sequences were chosen as a reference for two main reasons: (a) V. vinifera proteins appear to have a slower evolution rate than other dicots (Cenci et al. 2010; 2013; Yue et al. 2010), and (b) its genome experienced the lowest number of WGDs after the monocot/dicot lineage divergence (Bowers et al. 2003; Jaillon et al. 2007; Tang et al. 2010; D'Hont et al. 2012; Paterson et al. 2009) which is the probable main reason for its low number of NAC members.

Among the 103 A. thaliana NAC members, 19 displayed very low Blastp scores (lower than 170) with the best hit of the $V$. vinifera protein database and with the other two analysed species. Consequently, only the 84 NAC members (showing Blastp score higher than 250) were considered for the grouping. Similarly, 73 Oryza sativa NAC sequences showed very low similarity with NACs of other analysed species and were not considered in the orthologous grouping.

Finally, among the 167 M. acuminata NAC sequences, those five having a very low score with the best $V$. vinifera hit (less than 130) were discarded, and the remaining 162 with BLAST scores higher than 200 were retained for the orthologous grouping.

Thus, 40 OGs containing NAC members were obtained (Table 1). The nomenclature we used for OGs is based on the eight $V$. vinifera clusters (Wang et al. 2013) with a letter to distinguish different groups inside the clusters (Table 1). For example, among the nine $V$. vinifera NAC sequences included in cluster 1, eight OGs were named from 1a to $1 \mathrm{~h}$ (being two sequences, VvNAC05 and VvNAC11, included 
Table 1 List of the 40 OGs of the NAC gene family based on sequence analysis in four angiosperm species

\begin{tabular}{|c|c|c|c|c|c|}
\hline $\begin{array}{l}\text { Orthologous } \\
\text { group }\end{array}$ & $V$. vinifera & A. thaliana & O. sativa & M. acuminata & Function \\
\hline $1 \mathrm{a}$ & VvNAC56 & ANAC074 & $\begin{array}{l}\text { Os02g41450.1 } \\
\text { Os02g56600.1 } \\
\text { Os03g01870.1 } \\
\text { Os04g43560.1 } \\
\text { Os10g33760.1 }\end{array}$ & $\begin{array}{l}\text { Achr1T10860 } \\
\text { Achr1T20530 } \\
\text { Achr5T18670 } \\
\text { Achr6T36550 } \\
\text { Achr8T18980 } \\
\text { Achr11T00880 } \\
\text { Achr11T22760 } \\
\text { AchrUn_randomT17260 } \\
\text { AchrUn_randomT02760 }\end{array}$ & $\begin{array}{l}\text { MaNAC6 (Achr11T00880), } \\
\text { banana fruit ripening (Shan } \\
\text { et al. 2012) }\end{array}$ \\
\hline $1 b$ & VvNAC33 & $\begin{array}{l}\text { ANAC021/022 } \\
\text { ANAC_- } \\
\text { At3g12977.1 }\end{array}$ & $\begin{array}{l}\text { Os02g06950.1 } \\
\text { Os04g52810.1 } \\
\text { Os06g46270.1 } \\
\text { Os08g10080.1 } \\
\text { Os12g41680.1 }\end{array}$ & $\begin{array}{l}\text { Achr3T23360 } \\
\text { Achr5T00500 } \\
\text { Achr5T26640 } \\
\text { Achr6T30050 } \\
\text { Achr6T31350 } \\
\text { Achr8T07120 } \\
\text { Achr8T33310 } \\
\text { Achr9T10210 } \\
\text { Achr9T26140 } \\
\text { Achr11T25720 }\end{array}$ & $\begin{array}{l}\text { NAC1 (ANAC21/022) } \\
\text { Root development (Guo } \\
\text { et al. 2004); Os12g41680, } \\
\text { abiotic stresses (Nuruzza- } \\
\text { man et al. 2012); MaNAC5 } \\
\text { (Achr9T26140), banana fruit } \\
\text { ripening (Shan et al. 2012) }\end{array}$ \\
\hline $1 \mathrm{c}$ & VvNAC65 & ANAC038/039 & Os09g32260.1 & $\begin{array}{l}\text { Achr6T01770 } \\
\text { Achr7T19680 } \\
\text { Achr8T24680 }\end{array}$ & \\
\hline $1 d$ & VvNAC16 & $\begin{array}{l}\text { ANAC054 } \\
\text { ANAC098 }\end{array}$ & Os06g23650.1 & $\begin{array}{l}\text { Achr10T22350 } \\
\text { Achr10T26180 }\end{array}$ & $\begin{array}{l}\text { CUC1 and CUC2 (ANAC054 } \\
\text { and 098), shoot apical mer- } \\
\text { istem development (Takada } \\
\text { et al. 2001) }\end{array}$ \\
\hline $1 \mathrm{e}$ & VvNAC14 & ANAC031 & Os08g40030.1 & Achr9T20090 & $\begin{array}{l}\text { CUC3 (ANAC031), shoot } \\
\text { apical meristem development } \\
\text { (Hibara et al. 2006) }\end{array}$ \\
\hline 1f & VvNAC06 & ANAC058 & Os03g42630.1 & $\begin{array}{l}\text { Achr8T18420 } \\
\text { Achr9T00570 } \\
\text { Achr10T19900 }\end{array}$ & \\
\hline $1 \mathrm{~g}$ & VvNAC61 & $\begin{array}{l}\text { ANAC046 } \\
\text { ANAC087 }\end{array}$ & $\begin{array}{l}\text { Os01g01470.1 } \\
\text { Os01g29840.1 } \\
\text { Os03g21030.1 } \\
\text { Os07g48550.1 } \\
\text { Os11g03310.1 } \\
\text { Os11g03370.1 } \\
\text { Os12g03050.1 }\end{array}$ & $\begin{array}{l}\text { Achr3T09520 } \\
\text { Achr3T18020 } \\
\text { Achr5T07600 } \\
\text { Achr6T08600 } \\
\text { Achr6T32290 } \\
\text { Achr7T18330 } \\
\text { Achr8T21470 } \\
\text { Achr9T16920 } \\
\text { Achr10T05070 }\end{array}$ & $\begin{array}{l}\text { OsNAC45 (Os11g03370), } \\
\text { drought and salt toler- } \\
\text { ance (Zheng et al. 2009); } \\
\text { Os11g03370, Os12g03050, } \\
\text { virus infection (Nuruzzaman } \\
\text { et al. 2010) }\end{array}$ \\
\hline $1 \mathrm{~h}$ & $\begin{array}{l}\text { VvNAC05 } \\
\text { VvNAC11 }\end{array}$ & $\begin{array}{l}\text { ANAC059 } \\
\text { ANAC079/080 } \\
\text { ANAC092 } \\
\text { ANAC100 }\end{array}$ & $\begin{array}{l}\text { Os02g36880.1 } \\
\text { Os04g38720.1 }\end{array}$ & $\begin{array}{l}\text { Achr6T30570 } \\
\text { Achr7T11500 } \\
\text { Achr9T27530 }\end{array}$ & $\begin{array}{l}\text { AtNAC2 (ANAC059), salt } \\
\text { stress response and lateral } \\
\text { root development (He et al. } \\
\text { 2005); ANAC092, salt stress } \\
\text { (Balazadeh et al. 2010); } \\
\text { OsNAC2 (Os04g38720) Shoot } \\
\text { branching (Mao et al. 2007); } \\
\text { MaNAC3 (Achr9T27530), } \\
\text { banana fruit ripening (Shan } \\
\text { et al. 2012) }\end{array}$ \\
\hline $2 \mathrm{a}$ & $\begin{array}{l}\text { VvNAC02 } \\
\text { VvNAC22 }\end{array}$ & $\begin{array}{l}\text { ANAC007 } \\
\text { ANAC026 } \\
\text { ANAC101 }\end{array}$ & $\begin{array}{l}\text { Os02g42970.1 } \\
\text { Os04g45340.1 } \\
\text { Os06g01480.1 }\end{array}$ & $\begin{array}{l}\text { Achr6T36840 } \\
\text { Achr7T06640 } \\
\text { Achr8T11590 } \\
\text { Achr11T03780 } \\
\text { Achr11T17510 }\end{array}$ & $\begin{array}{l}\text { VND4-6 (ANAC007, 026, 101), } \\
\text { vascular development (Kubo } \\
\text { 2005) }\end{array}$ \\
\hline
\end{tabular}


Table 1 continued

\begin{tabular}{|c|c|c|c|c|c|}
\hline $\begin{array}{l}\text { Orthologous } \\
\text { group }\end{array}$ & V. vinifera & A. thaliana & O. sativa & M. acuminata & Function \\
\hline$\overline{2 b}$ & VvNAC23 & $\begin{array}{l}\text { ANAC037 } \\
\text { ANAC076 } \\
\text { ANAC105 }\end{array}$ & $\begin{array}{l}\text { Os03g03540.1 } \\
\text { Os10g38834.1 }\end{array}$ & $\begin{array}{l}\text { Achr8T12100 } \\
\text { Achr11T03040 }\end{array}$ & $\begin{array}{l}\text { VND1-3 (ANAC037, 076,105), } \\
\text { vascular development (Kubo } \\
\text { 2005); Os10g38834, drought } \\
\text { stress (Nuruzzaman et al. } \\
\text { 2012) }\end{array}$ \\
\hline $2 \mathrm{c}$ & VvNAC63 & ANAC030 & $\begin{array}{l}\text { Os04g59470.1 } \\
\text { Os08g01330.1 }\end{array}$ & $\begin{array}{l}\text { Achr3T22360 } \\
\text { Achr7T23170 }\end{array}$ & $\begin{array}{l}\text { VND7 (ANAC030), vascular } \\
\text { development (Kubo 2005) }\end{array}$ \\
\hline $2 \mathrm{~d}$ & VvNAC70 & $\begin{array}{l}\text { ANAC015 } \\
\text { ANAC070 }\end{array}$ & $\begin{array}{l}\text { Os04 g (unannotated, } \\
\text { OsI_35493) }\end{array}$ & $\begin{array}{l}\text { Achr2T05640 } \\
\text { Achr6T28890 }\end{array}$ & $\begin{array}{l}\text { Bearskin1-2 (ANAC015, 070), } \\
\text { Root cap maturation (Bennett } \\
\text { et al. 2010) }\end{array}$ \\
\hline $2 \mathrm{e}$ & VvNAC68 & ANAC033 & $\begin{array}{l}\text { Os02g15340.1 } \\
\text { Os06g33940.1 }\end{array}$ & $\begin{array}{l}\text { Achr2T20020 } \\
\text { Achr10T14400 } \\
\text { Achr10T21750 }\end{array}$ & $\begin{array}{l}\text { Sombrero (ANAC033), Root } \\
\text { cap maturation (Willemsen } \\
\text { et al. 2008) }\end{array}$ \\
\hline $2 \mathrm{f}$ & $\begin{array}{l}\text { VvNAC24 } \\
\text { VvNAC49 }\end{array}$ & $\begin{array}{l}\text { ANAC043 } \\
\text { ANAC066 } \\
\text { ANAC012 }\end{array}$ & $\begin{array}{l}\text { Os06g04090.1 } \\
\text { Os08g02300.1 }\end{array}$ & $\begin{array}{l}\text { Achr3T12230 } \\
\text { Achr7T05980 } \\
\text { Achr9T24450 } \\
\text { AchrUn_randomT21980 }\end{array}$ & $\begin{array}{l}\text { NST1-2 (ANAC043-066) and } \\
\text { SND1 (ANAC012), Second- } \\
\text { ary wall thickening (Mitsuda } \\
\text { et al. 2005; 2007) }\end{array}$ \\
\hline $3 a$ & $\begin{array}{l}\text { VvNAC08 } \\
\text { VvNAC39 }\end{array}$ & $\begin{array}{l}\text { ANAC002 } \\
\text { ANAC032 } \\
\text { ANAC081 } \\
\text { ANAC102 }\end{array}$ & $\begin{array}{l}\text { Os01g66120.1 } \\
\text { Os05g34830.1 } \\
\text { Os11g08210.1 }\end{array}$ & $\begin{array}{l}\text { Achr3T18990 } \\
\text { Achr6T17720 } \\
\text { Achr6T18720 } \\
\text { Achr6T25380 } \\
\text { Achr7T23250 } \\
\text { Achr10T04570 }\end{array}$ & $\begin{array}{l}\text { ATAF1 (ANAC002) Drought } \\
\text { stress responses (Hu } \\
\text { et al. 2006); ATAF1-2 } \\
\text { (ANAC002-081), repres- } \\
\text { sor of pathogenesis-related } \\
\text { proteins (Delessert et al. 2005; } \\
\text { Wang et al. 2009); OsNAC6 } \\
\text { (SNAC2), 5, 52 (Os01g66120, } \\
\text { Os11g08210, Os5g34830) } \\
\text { Abiotic stress (Ohnishi et al. } \\
\text { 2005; Nakashima et al. 2007; } \\
\text { Hu et al. 2008: Takasaki et al. } \\
\text { 2010; Gao et al. 2009) }\end{array}$ \\
\hline $3 b$ & VvNAC44 & & $\begin{array}{l}\text { Os01g60020.1 } \\
\text { Os03g60080.1 } \\
\text { Os07g12340.1 }\end{array}$ & $\begin{array}{l}\text { Achr4T02390 } \\
\text { Achr4T10310 } \\
\text { Achr5T07590 } \\
\text { Achr6T32330 } \\
\text { Achr7T21780 } \\
\text { Achr10T29200 }\end{array}$ & $\begin{array}{l}\text { OsNAC19 (0s3g60080), } \\
\text { response to infection by } \\
\text { M. grisea (Lin et al. 2007); } \\
\text { SNAC1 (Os3g60080) drought } \\
\text { stress (Hu et al. 2006 }\end{array}$ \\
\hline $3 \mathrm{c}$ & $\begin{array}{l}\text { VvNAC60 } \\
\text { VvNAC26 }\end{array}$ & $\begin{array}{l}\text { ANAC047 } \\
\text { ANAC029 }\end{array}$ & $\begin{array}{l}\text { Os01g01430.1 } \\
\text { Os03g21060.1 } \\
\text { Os05g34310.1 } \\
\text { Os07g48450.1 } \\
\text { Os11g03300.1 } \\
\text { Os12g03040.1 }\end{array}$ & $\begin{array}{l}\text { Achr3T18010 } \\
\text { Achr4T02380 } \\
\text { Achr6T32320 } \\
\text { Achr7T21770 } \\
\text { Achr9T04960 } \\
\text { Achr10T12860 } \\
\text { AchrUn_randomT17360 }\end{array}$ & $\begin{array}{l}\text { AtNAP (ANAC029), leaf senes- } \\
\text { cence (Guo and Gan 2006); } \\
\text { OsNAC10 (Os11g03300) } \\
\text { Drought tolerance (Jeong } \\
\text { et al. 2010); Os11g03300, } \\
\text { Os12g03040, reponse to Mag- } \\
\text { naporte grisea infection, (Sun } \\
\text { et al. 2013) }\end{array}$ \\
\hline $3 \mathrm{~d}$ & $\begin{array}{l}\text { VvNAC03 } \\
\text { VvNAC43 }^{\mathrm{a}} \\
\text { VvNAC18 }\end{array}$ & $\begin{array}{l}\text { ANAC018 } \\
\text { ANAC025 } \\
\text { ANAC056 }\end{array}$ & Os07g37920.1 & $\begin{array}{l}\text { Achr1T08860 } \\
\text { Achr9T19520 }\end{array}$ & $\begin{array}{l}\text { Os07g37920, senescence } \\
\text { (Distelfeld et al. 2012) }\end{array}$ \\
\hline $3 e$ & VvNAC17 & $\begin{array}{l}\text { ANAC072 } \\
\text { ANAC019 } \\
\text { ANAC055 }\end{array}$ & & & $\begin{array}{l}\text { ANAC072 (RD26), } 019 \text { and } \\
\text { 055, Drought tolerance (Tran } \\
\text { et al. 2004); ANAC019, } \\
\text { ANAC055, Defense disease, } \\
\text { Jasmonate pathway (Bu et al. } \\
\text { 2008) }\end{array}$ \\
\hline $4 a$ & VvNAC64 & $\begin{array}{l}\text { ANAC028 } \\
\text { ANAC045 } \\
\text { ANAC086 }\end{array}$ & Os03g02800.1 & $\begin{array}{l}\text { Achr1T02820 } \\
\text { Achr8T13430 } \\
\text { Achr11T01400 } \\
\text { Achr11T16860 } \\
\text { AchrUn_randomT08190 }\end{array}$ & $\begin{array}{l}\text { RIM1 (Os3g02800) virus resist- } \\
\text { ance; Jasmonate pathway } \\
\text { signalling (Yoshii et al. 2010) }\end{array}$ \\
\hline
\end{tabular}


Table 1 continued

\begin{tabular}{|c|c|c|c|c|c|}
\hline $\begin{array}{l}\text { Orthologous } \\
\text { group }\end{array}$ & V. vinifera & A. thaliana & O. sativa & M. acuminata & Function \\
\hline$\overline{4 b}$ & VvNAC57 & ANAC057 & $\begin{array}{c}\text { Os09g38000.1 } \\
\text { Os09g38010.1 }\end{array}$ & $\begin{array}{l}\text { Achr3T13880 } \\
\text { Achr9T23940 }\end{array}$ & \\
\hline $4 c$ & VvNAC13 & $\begin{array}{l}\text { ANAC071 } \\
\text { ANAC011 } \\
\text { ANAC096 }\end{array}$ & Os10g42130.1 & $\begin{array}{l}\text { Achr3T18070 } \\
\text { Achr5T03360 } \\
\text { Achr5T18140 } \\
\text { Achr11T17780 }\end{array}$ & \\
\hline $4 d$ & $\begin{array}{l}\text { VvNAC20 } \\
\text { VvNAC } 21\end{array}$ & $\begin{array}{l}\text { ANAC050 } \\
\text { ANAC051/052 } \\
\text { ANAC053 } \\
\text { ANAC077 } \\
\text { ANAC078 }\end{array}$ & $\begin{array}{l}\text { Os02g57650.1 } \\
\text { Os08g44820.1 }\end{array}$ & $\begin{array}{l}\text { Achr2T04010 } \\
\text { Achr9T20400 } \\
\text { Achr9T23580 } \\
\text { AchrUn_randomT07620 }\end{array}$ & \\
\hline $4 \mathrm{e}$ & VvNAC48 & $\begin{array}{l}\text { ANAC082 } \\
\text { ANAC103 }\end{array}$ & $\begin{array}{l}\text { Os05g35170.1 } \\
\text { Os_AK068153 }\end{array}$ & $\begin{array}{l}\text { Achr7T23330 } \\
\text { Achr10T04530 } \\
\text { Achr11T09000 }\end{array}$ & $\begin{array}{l}\text { VNI1 (ANAC082), vascular } \\
\text { development (Yamaguchi } \\
\text { et al. 2010) }\end{array}$ \\
\hline $4 \mathrm{f}$ & VvNAC69 & ANAC020 & & & \\
\hline $4 \mathrm{~g}$ & VvNAC15 & $\begin{array}{l}\text { ANAC013 } \\
\text { ANAC016 } \\
\text { ANAC017 }\end{array}$ & Os09g32040.1 & $\begin{array}{l}\text { Achr8T24280 } \\
\text { AchrUn_randomT11980 }\end{array}$ & \\
\hline $5 \mathrm{a}$ & $\begin{array}{l}\text { VvNAC01 } \\
\text { VvNAC07 } \\
\text { VvNAC73 }\end{array}$ & $\begin{array}{l}\text { ANAC041 } \\
\text { ANAC084 } \\
\text { ANAC097 } \\
\text { ANAC083 }\end{array}$ & $\begin{array}{l}\text { Os01g70110.1 } \\
\text { Os08g42400.1 } \\
\text { Os09g33490.1 } \\
\text { Os11g31330.1 } \\
\text { Os12g29330.1 }\end{array}$ & $\begin{array}{l}\text { Achr2T21110 } \\
\text { Achr3T18680 } \\
\text { Achr6T16560 } \\
\text { Achr6T20870 } \\
\text { Achr6T23840 } \\
\text { Achr7T22480 } \\
\text { Achr10T16940 } \\
\text { Achr10T11910 } \\
\text { AchrUn_randomT24680 }\end{array}$ & $\begin{array}{l}\text { VNI2 (ANAC083), vascuolar } \\
\text { development, salt tolerance, } \\
\text { leaf senescence (Yamaguchi } \\
\text { et al. 2010; Yang et al. 2011; } \\
\text { Seo and Park 2011) }\end{array}$ \\
\hline $5 b$ & $\begin{array}{l}\text { VvNAC25 } \\
\text { VvNAC51 } \\
\text { VvNAC52 } \\
\text { VvNAC53 } \\
\text { VvNAC54 } \\
\text { VvNAC55 } \\
\text { VvNAC71 }\end{array}$ & $\begin{array}{l}\text { ANAC014 } \\
\text { ANAC062 } \\
\text { ANAC091 } \\
\text { ANAC } \\
\text { At4g35580 }\end{array}$ & $\begin{array}{l}\text { Os06g01230.1 } \\
\text { Os08g06140.1 }\end{array}$ & $\begin{array}{l}\text { Achr2T11810 } \\
\text { Achr3T00330 } \\
\text { Achr4T29170 } \\
\text { Achr11T07800 } \\
\text { AchrUn_randomT04060 }\end{array}$ & $\begin{array}{l}\text { TIP (ANAC091), virus interact- } \\
\text { ing (Ren et al. 2000); NTL6 } \\
\text { (ANAC062) drought stress } \\
\text { (Kim et al. 2012); ntm2 } \\
\text { (ANAC069) salt stress (Park } \\
\text { et al. 2011); NTL9 (ANAC_- } \\
\text { At4g35580) salt stress (Yoon } \\
\text { et al. 2008) }\end{array}$ \\
\hline $5 c+M S$ & VvNAC67 & $\begin{array}{l}\text { ANAC040 } \\
\text { ANAC060 } \\
\text { ANAC089 }\end{array}$ & Os01g15640.1 & $\begin{array}{l}\text { Achr5T23620 } \\
\text { Achr6T03200 }\end{array}$ & $\begin{array}{l}\text { NTL8 (ANAC040), regulation } \\
\text { of salt-responsive flowering } \\
\text { (Kim et al. 2007) }\end{array}$ \\
\hline $6 \mathrm{a}$ & VvNAC40 & ANAC034 & $\begin{array}{l}\text { Os01g66490.1 } \\
\text { Os05g34600.1 } \\
\text { Os08g02160.1 }\end{array}$ & $\begin{array}{l}\text { Achr7T23650 } \\
\text { Achr3T21690 } \\
\text { Achr5T17060 } \\
\text { Achr10T04320 } \\
\text { Achr10T10690 } \\
\text { Achr11T08970 } \\
\text { Achr11T22590 } \\
\text { Achr11T26450 }\end{array}$ & $\begin{array}{l}\text { LOV1 (ANAC034) Cold } \\
\text { response, photoperiod path- } \\
\text { way (Yoo et al. 2007) }\end{array}$ \\
\hline $6 b$ & $\begin{array}{l}\text { VvNAC10 } \\
\text { VvNAC27 }\end{array}$ & $\begin{array}{l}\text { ANAC009 } \\
\text { ANAC094 }\end{array}$ & $\begin{array}{l}\text { Os08g33910.1 } \\
\text { Os02g51120.1 }\end{array}$ & $\begin{array}{l}\text { Achr6T02680 } \\
\text { Achr10T08120 }\end{array}$ & $\begin{array}{l}\text { FEZ (ANAC009), Root cap } \\
\text { maturation (Willemsen } \\
\text { et al. 2008); ONAC063 } \\
\text { (Os08g33910) salt stress } \\
\text { (Yokotani et al. 2009) }\end{array}$ \\
\hline
\end{tabular}


Table 1 continued

\begin{tabular}{|c|c|c|c|c|c|}
\hline $\begin{array}{l}\text { Orthologous } \\
\text { group }\end{array}$ & V. vinifera & A. thaliana & O. sativa & M. acuminata & Function \\
\hline$\overline{6 c}$ & $\begin{array}{l}\text { VvNAC28 } \\
\text { VvNAC29 } \\
\text { VvNAC30 } \\
\text { VvNAC31 } \\
\text { VvNAC32 } \\
\text { VvNAC36 }\end{array}$ & $\begin{array}{l}\text { ANAC }_{-} \\
\text {At3g12910.1 } \\
\text { ANAC042 }\end{array}$ & $\begin{array}{l}\text { Os03g56580.1 } \\
\text { Os07g04560.1 } \\
\text { Os12g43530.1 }\end{array}$ & $\begin{array}{l}\text { Achr4T23030 } \\
\text { Achr4T32010 } \\
\text { Achr5T02170 } \\
\text { Achr6T31585 } \\
\text { Achr7T00860 } \\
\text { Achr9T10040 } \\
\text { Achr10T08420 }\end{array}$ & $\begin{array}{l}\text { ANAC042, heat stress } \\
\text { (Shahnejat-Bushehri et al. } \\
\text { 2012) and pathogen infection } \\
\text { (Saga et al. 2012); MaNAC2 } \\
\text { (Achr6T31585), MaNAC4 } \\
\text { (Achr7T00860), banana fruit } \\
\text { ripening (Shan et al. 2012); } \\
\text { Os10g38834, drought stress } \\
\text { (Nuruzzaman et al. 2012) }\end{array}$ \\
\hline $7 \mathrm{a}$ & $\begin{array}{l}\text { VvNAC34 } \\
\text { VvNAC37 }\end{array}$ & $\begin{array}{l}\text { ANAC010 } \\
\text { ANAC073 }\end{array}$ & $\begin{array}{l}\text { Os01g48130.1 } \\
\text { Os05g48850.1 }\end{array}$ & $\begin{array}{l}\text { Achr2T09080 } \\
\text { Achr4T02730 } \\
\text { Achr6T27000 }\end{array}$ & $\begin{array}{l}\text { MaNAC1 (Achr6T27000), } \\
\text { banana fruit ripening (Shan } \\
\text { et al. 2012) }\end{array}$ \\
\hline $7 \mathrm{~b}$ & VvNAC19 & $\begin{array}{l}\text { ANAC075 } \\
\text { ANAC099 }\end{array}$ & $\begin{array}{l}\text { Os01g09550.1 } \\
\text { Os05g10620.1 } \\
\text { Os06g } 36480.1\end{array}$ & $\begin{array}{l}\text { Achr2T16590 } \\
\text { Achr4T30940 } \\
\text { Achr6T05480 } \\
\text { Achr7T09510 } \\
\text { Achr10T27600 }\end{array}$ & \\
\hline $7 \mathrm{c}$ & VvNAC12 & ANAC008 & Os06g15690.1 & $\begin{array}{l}\text { Achr3T07330 } \\
\text { Achr6T11230 }\end{array}$ & $\begin{array}{l}\text { SOG1 (ANAC008), response to } \\
\text { gamma radiation (Yoshiyama } \\
\text { et al. 2009); Os06g15690 } \\
\text { drought stress (Nuruzzaman } \\
\text { et al. 2012) }\end{array}$ \\
\hline $7 \mathrm{~d}$ & VvNAC59 & $\begin{array}{l}\text { ANAC044 } \\
\text { ANAC085 }\end{array}$ & $\begin{array}{l}\text { Os04g40140.1 } \\
\text { Os02g38130.1 }\end{array}$ & $\begin{array}{l}\text { Achr9T01880 } \\
\text { AchrUn_randomT17050 }\end{array}$ & $\begin{array}{l}\text { Os04g477300 (Os04g40140) } \\
\text { boron-toxicity toler- } \\
\text { ance (Ochiai et al. 2011); } \\
\text { Os0238130, viral infection } \\
\text { (Nuruzzaman et al. 2010) }\end{array}$ \\
\hline $7 \mathrm{e}$ & $\begin{array}{l}\text { VvNAC47 } \\
\text { VvNAC58 }\end{array}$ & ANAC104 & $\begin{array}{l}\text { Os02g34970.1 } \\
\text { Os04g35660.1 }\end{array}$ & $\begin{array}{l}\text { Achr6T17670 } \\
\text { Achr6T18640 } \\
\text { Achr6T25790 } \\
\text { Achr10T10790 }\end{array}$ & $\begin{array}{l}\text { XND1 (ANAC104), lignocel- } \\
\text { lulose synthesis (Zhao et al. } \\
\text { 2007); Os02g34970) drought } \\
\text { stress; viral infection (Nuruz- } \\
\text { zaman et al. 2010; 2012) }\end{array}$ \\
\hline $7 f$ & VvNAC09 & & Os10g21560.1 & $\begin{array}{l}\text { Achr2T06610 } \\
\text { Achr4T07148 } \\
\text { Achr7T26050 }\end{array}$ & \\
\hline $8 \mathrm{a}$ & $\begin{array}{l}\text { VvNAC04 } \\
\text { VvNAC41 }\end{array}$ & ANAC036 & $\begin{array}{l}\text { Os03g04070.1 } \\
\text { Os06g51070.1 }\end{array}$ & $\begin{array}{l}\text { Achr1T02710 } \\
\text { Achr3T00560 } \\
\text { Achr3T14720 } \\
\text { Achr5T19060 } \\
\text { Achr7T04030 } \\
\text { Achr11T01320 } \\
\text { AchrUn_randomT08220 }\end{array}$ & \\
\hline $8 b$ & $\begin{array}{l}\text { VvNAC46 } \\
\text { VvNAC62 } \\
\text { VvNAC74 }\end{array}$ & $\begin{array}{l}\text { ANAC061 } \\
\text { ANAC090 }\end{array}$ & $\begin{array}{l}\text { Os01g64310.1 } \\
\text { Os05g37080.1 } \\
\text { Os11g05614.1 } \\
\text { Os11g45950.1 } \\
\text { Os12g05990.1 }\end{array}$ & $\begin{array}{l}\text { Achr6T19400 } \\
\text { Achr8T01410 } \\
\text { Achr9T29750 } \\
\text { Achr10T04720 }\end{array}$ & $\begin{array}{l}\text { Os11g05614, virus infection } \\
\text { (Nuruzzaman et al. 2010) }\end{array}$ \\
\hline
\end{tabular}

The last column contains information on known functions for genes of the relative groups. Sequences showing best reciprocal Blastp hits are indicated in bold. Os08g33670.1 and Os11g03310.1 were close to the sequences of cluster 1, but they could not be assigned to a specific group. VvNAC42 and VvNAC45 could not be resolved between groups 3c or 3d

a Putative pseudogenes

b Os11g03310.1 was assigned to the group $1 \mathrm{~g}$ based on the phylogenetic analysis results

in the OG $1 \mathrm{~h}$ ). Similarly, cluster 5 (containing eleven $V$. vinifera NAC sequences) was subdivided into three OGs: 5a (containing VvNAC01, VvNAC07 and VvNAC73), 5b
(VvNAC25, VvNAC51-VvNAC55 and VvNAC71) and 5c (VvNAC67). Two of these groups did not contain any $A$. thaliana sequence $(3 \mathrm{~b}, 7 \mathrm{f})$, whereas three other groups did 
not contain any monocot sequences $(3 \mathrm{e}, 4 \mathrm{f}$, and $5 \mathrm{c})$. An additional OG was obtained with one $O$. sativa and two $M$. acuminata very closely related sequences; this monocotspecific group was named MS (Table 1). Four sequences (Os08g33670.1 and Os11g03310.1 close to the sequences of OGs 1; VvNAC42 and VvNAC45 close to OGs 3c or $3 d)$ could not be assigned due to very similar Blastp scores with sequences of different OGs.

\section{OrthoMCL analysis}

To verify whether our orthologous inferences could be reproduced automatically in such a very large gene family as the NAC, OG sequences were submitted to OrthoMCL clustering, using a panel of growing inflation parameters (from 1.5 to 14) that increase the cluster stringency. With the lowest stringency, six clusters were obtained (Fig. 2 and Online Resources 5) containing NAC members of variable numbers (from 4 to 10) of those OGs determined by expert comparison. None of these OGs was split in different OrthoMCL clusters. The increase in stringency did not allow OG differentiation, but with higher inflation parameters, single NAC sequences were isolated or OGs split in different clusters (Fig. 2, Online Resources 6-10). Only the cluster containing all NAC sequences of OGs $7 \mathrm{a}-\mathrm{d}$ was maintained regardless of stringency level (Fig. 2, Online Resources 5-10).

\section{Structural analysis of NAC genes}

In addition to the sequence similarity, the exon/intron structure of the NAC genes was comprehensively analysed. Almost all NAC genes of OGs 1, 2, 3, 6 and 8 were found to have very similar structures, with three exons that aligned well (Fig. 3). The first exon contains A and B subdomains (Kikuchi et al. 2000) and ends at the first nucleotide of the first codon after the B subdomain; the second exon contains the $\mathrm{C}$ and $\mathrm{D}$ subdomains and ends at the third nucleotide of a codon; the third exon begins with the $\mathrm{E}$ subdomain and contains all the $\mathrm{C}$-terminal region of the gene that includes the TRR. A few exceptions to this typical structure were observed in A. thaliana and $O$. sativa due to intron loss or, in other words, exon merging. In A. thaliana, only ANAC066 and ANAC101 genes are composed of two exons, because the second intron has been lost. In O. sativa, Os06g23650.1, Os08g40030.1 and Os11g05614.1 lost the first intron, Os03g42630.1, Os01g01470.1 and Os01g29840.1 lost the second intron, and Os12g05990.1 and Os06g51070.1 lost both introns. Moreover, all the three NAC members of the $3 b$ group lost the first intron, whereas only the Os07g12340.1 gene lost also the second intron resulting in a mono-exonic structure.
Genes included in OGs 4, 5 and 7 have more variable numbers of exons. OGs $4 \mathrm{a}-\mathrm{h}$ and $5 \mathrm{a}-\mathrm{c}$ have the typical position for first two introns, but additional introns could be present in the TRR, varying from none (OGs $4 b$ and $5 a$ ) to four (OG $5 \mathrm{~b}$ ). In monocot sequences of OG $4 \mathrm{~d}$, the first intron was lost and, consequently, the first exon contains the first four subdomains (Fig. 3).

OGs $7 \mathrm{a}$-d have particular features. E subdomains are not recognizable and the beginning of the gene has a different structure. OGs 7a-b genes have an additional exon at the beginning, so A-B subdomains are included in the second exon (that ends at the same position as the first exon of other groups), and third exon contains the C-D subdomains (Fig. 3). These two OGs can be differentiated according to their structure. Genes of OG 7a have only three exons; in OG $7 \mathrm{~b}$, genes are longer and have three additional exons in the TRR. Genes in OGs 7c and 7d have two additional exons at the beginning, the first with a very small coding region (3-4 amino acids). Consequently the A-B subdomains are in the third exon, whereas the $\mathrm{C}$-D subdomains are in the fourth one. The fifth and sixth exons contain the TRR (only Os04g40140.1 has 7 exons due to an additional intron in the fourth exon). Finally, genes of OG 7e have the typical structure with three exons and genes of group $7 \mathrm{f}$ have two additional exons located in the TRR (with the exception of GSMUA_Achr2T06610.1 that merged the last two exons).

Phylogenetic analyses

A phylogenetic analysis was performed with all the sequences included in the OGs $(84,69,92$ and 162 for $A$. thaliana, $V$. vinifera, O. sativa and M. acuminata, respectively) and a gene tree was obtained (Fig. 4 and Online Resources 11) based on 86 aligned amino acids, all belonging to the NAC domain. Twenty-six clusters were observed that contain all the NAC members of the OGs determined by expert comparison. On the other hand, a number of sequences relationships were not well defined. Two OGs, MS and 5c (specific to monocots and dicots, respectively), were consistent with the phylogenetic results, but these clusters were closely related and formed a unique cluster $($ aLRT $=0.876)$. Consequently these two OGs were merged into the $5 \mathrm{c}$ OG for subsequent analysis.

In order to verify whether the phylogenetic resolution could be improved using a set of more closely related sequences, we performed additional phylogenetic analyses with the sequences of OGs $2 \mathrm{a}-\mathrm{f}$ (51 sequences) and $7 \mathrm{a}-\mathrm{d}$ (33 sequences) which, in the global NAC gene tree, are clustered and sharply isolated from all other NAC but not well resolved in clear sub-clusters (Online Resources 11). The trees were obtained based on 163 and 175 aligned amino acids, respectively, and they contained also positions 
Fig. 2 Comparison between expert versus OrthoMCL automatic analysis for OG detection. Clusters obtained by OrthoMCL with three increasing inflation parameter and the OG member included in each cluster

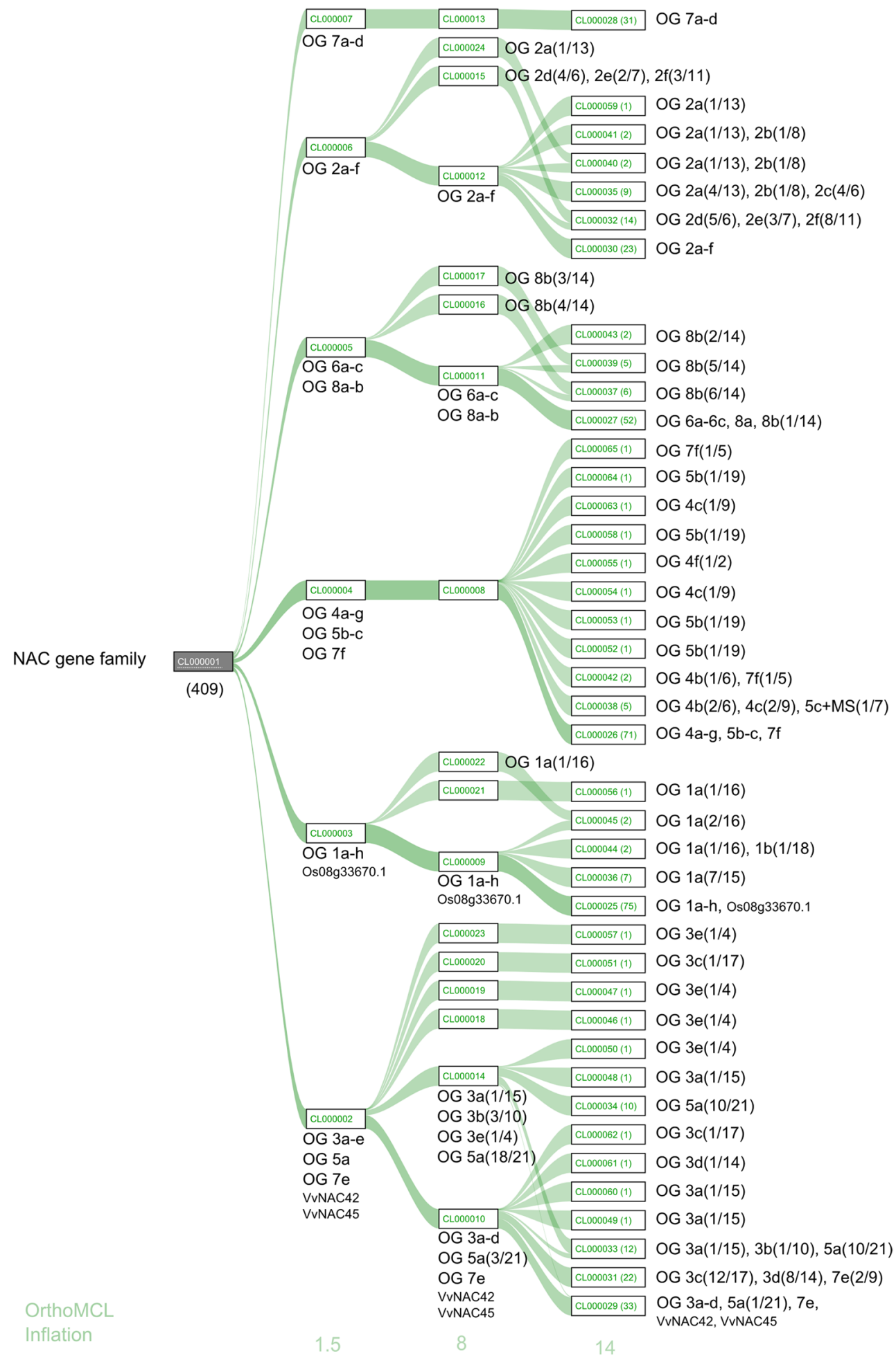

outside the NAC domain. The phylogenetic trees were perfectly consistent with expertly determined OGs, being all the sequences in OG-specific clusters, with aLRT values spanning between 0.790 and 0.997 (Online Resources 12).

Finally, phylogenetic analyses were performed for each expertly identified OG; since A. thaliana or monocots
NAC sequences were lacking in four OGs $(3 \mathrm{~b}, 3 \mathrm{e}, 4 \mathrm{f}$ and $7 f$ ), only thirty-six trees were built (Online Resources 13). Among the 36 phylogenetic trees obtained, 31 showed the expected dicot and monocot clustering. In two additional trees, a sequence of $M$. acuminata (Achr7T17500, OG $1 \mathrm{~h}$ ) and two sequences of $A$. thaliana (ANAC084 and 
Fig. 3 Exon/intron structure of NAC genes according to the orthologous groups

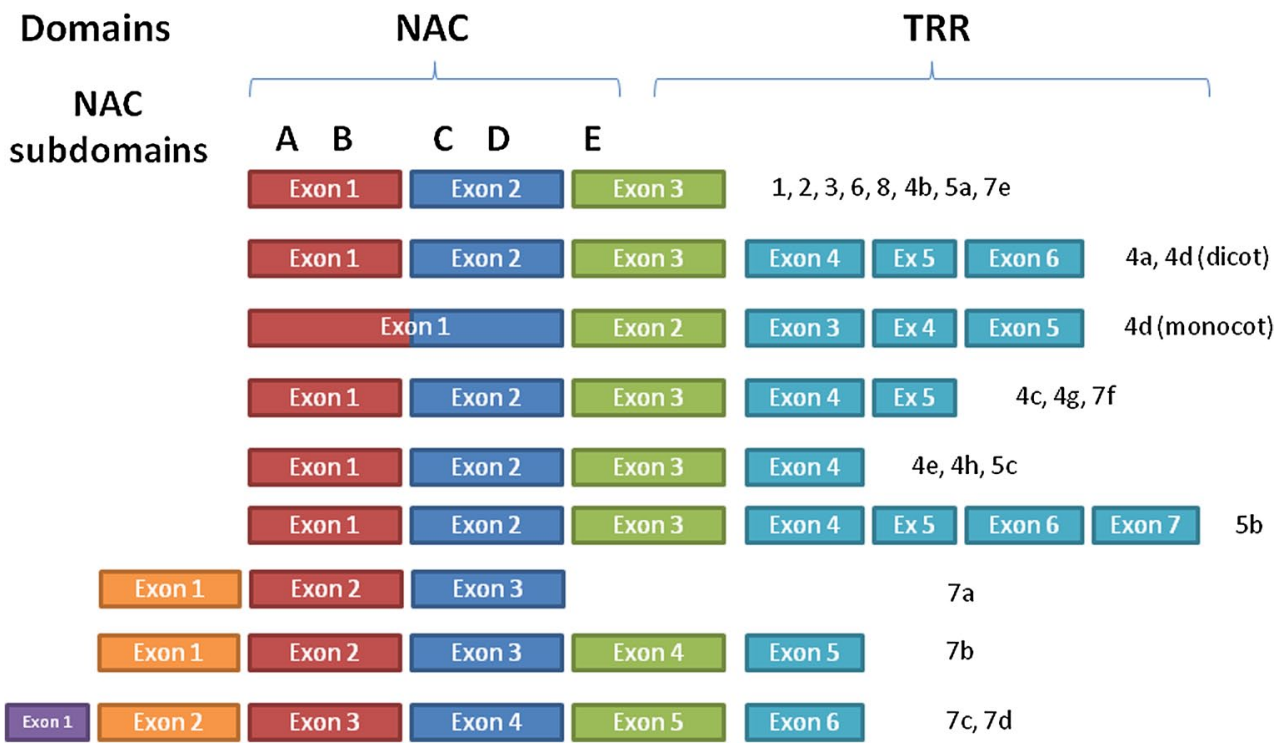

Fig. 4 Maximum Likelihood phylogenetic tree of NAC proteins. Phylogenetic analysis was carried with protein sequences from A. thaliana (ARATH), V. vinifera (VITVI), O. sativa (ORYSA) and M. acuminata (MUSAC) as described in the "Methods". Branch support values correspond to approximate likelihood ratio test (a-LRT) results. The 26 clusters supporting the grouping were indicated on the figure with the numbering proposed in this work. Leaf colors of the gene tree are colored according to their species. PhyloXML format of this gene tree and phylogenetic trees of the groups are provided as Online Resources 4

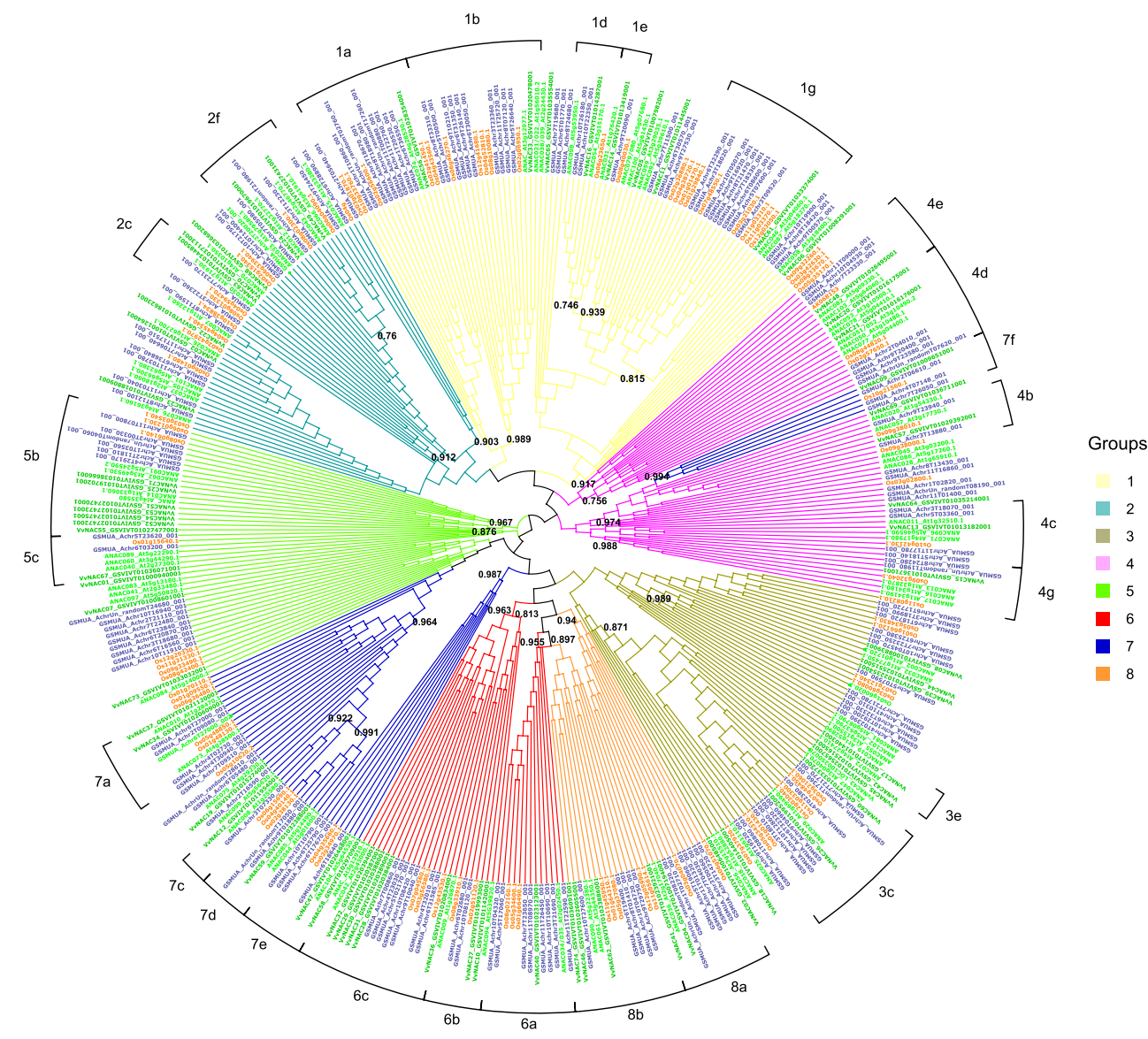

ANAC097, OG 5a) occupy unexpected positions; all these three sequences were characterized by very long branches (Online Resources 13). In the remaining three trees, one was unresolved (3c) whereas in the other two (3d and 1d) monocot and dicot sequences were not separated into phylum-specific clusters. For a given species, when more than one sequence is included in an OG, species-specific clusters are often observed, which indicates gene amplifications took place after the lineage divergence. On the other hand, in few cases sub-clusters containing dicot and/or 
monocots (e.g. group 2a, Online Resources 13) could indicate gene duplications that occurred before the Arabidopsis-Vitis and/or Musa-Oryza lineage divergence.

\section{Discussion}

The phylogenetic position of $M$. acuminata (order Zingiberales, a sister group to the well-studied Poales order) offers the opportunity for a deeper exploration into monocot evolution and into plant genome evolution in general. The present study analysed the evolution of NAC genes, a high copy TF family in the monocots and dicots, the two major lineages of angiosperms.

\section{Musa acuminata NAC}

The availability of the $M$. acuminata genome sequence allows global analysis of large gene families such as NAC transcription factors. Automatic gene annotation provides an initial panorama of abundance and chromosome distribution of the members of this gene family. As automatic gene structure annotation can be imprecise, we performed a revision of the members of this large family. We considered 167 genes to be potentially functional, and eliminated from the analyses five pseudogenes predicted by the presence of mutations modifying the typical gene structure and by the lack of observed transcription. However, due to the limited availability of transcriptome data, the lack of functionality of these genes needs to be confirmed, as well as the effective activities of the potentially functional genes. Compared to other species, the M. acuminata genome contains a high number of NAC genes. Twelve ancestral genomic regions pre-dating the last two WGDs could be reconstructed by analysis of gene collinearity among Musa chromosomes (D'Hont et al. 2012). When the NAC genes were positioned in these ancestral groups, at least 43 genes could be inferred as derived from duplications during one of the last two Musa WGDs.

Orthologous grouping of NAC genes from different species

The orthologous grouping of NAC sequences belonging to four distinctly divergent species was based on similarities inferred by Blastp analysis. Even if Blastp does not provide a distance measure between two sequences, a better Blastp score was assumed to indicate a greater similarity between compared sequences.

Among all the NAC sequences available, a number of sequences showed a very low Blastp score with any NAC of other species. The Blastp score is influenced by the distance between the protein sequences, which in turn is influenced by the genetic distance between the analysed species. The latter in turn is determined by the phylogenetic position and evolution rate of the analysed species. For example, a lower genetic distance is expected between two monocot species than between a monocot and a dicot. Given this, empirically set thresholds of Blastp scores were used to exclude highly divergent sequences from the comparative analysis. Moreover, these highly divergent sequences showed similar Blastp scores with sequences assigned to several OGs that render these sequences unassignable to any OG. Sequences showing low Blastp scores with other species could be the result of speciesspecific sequence evolution or degradation due to the relaxation of any purifying selective pressure (pseudogenization).

The existence of a large group of $O$. sativa-specific NACs was highlighted in the study of Nuruzzaman et al. (2010). The large majority of these sequences resulted in cluster II in the phylogenetic analysis of Fang et al. (2008). In the light of their species specificity, these sequences were not considered in our comparative study.

More than 400 sequences were manually compared for their similarity with a species by species Blastp analysis. Forty OGs containing NAC sequences from at least two species were obtained. In addition to their being lower in number, NAC sequences of $V$. vinifera were chosen as the reference for comparison because of the reported lower evolution rate of this species compared to other dicots (Cenci et al. 2010; 2013; Yue et al. 2010). The phylogenetic analyses performed on NAC sequences of 36 OGs were consistent with these reports, i.e. the branch lengths of NAC sequences being generally shorter in $V$. vinifera than in A. thaliana (Online Resources 13).

Using the full set of sequences, phylogenetic analyses confirmed most of the orthologous grouping results: 26 of the 40 OGs were found consistent with clustering, i.e. all the NACs of a given OG were found in an OG-specific cluster. The other groups could not be resolved in OGspecific clusters, although no inconsistencies were noted with the proposed grouping (i.e. no well-supported clusters containing sequences from different OGs were observed). OGs 7a-d appeared very divergent from the other NACs, which is consistent with their particular structure (additional exons at the beginning) and sequence (lack of E subdomains). The NAC of the OGs $2 \mathrm{a}-\mathrm{f}$ and $7 \mathrm{a}-\mathrm{d}$ resulted in two well-defined clusters, but the distribution of NAC in specific OGs was not completely resolved. By performing phylogenetic analysis restricted to the sequences of these OGs, we obtained perfectly consistent clusters with the grouping made by the Blastp reciprocal analysis. The number of amino acid positions in the filtered multiple alignment was approximately twice the number of the ones obtained with the whole set of NAC sequences (86 amino acids). It is likely that the phylogenetic signal was clearer and enabled a better resolution of these clusters than the global analysis. 
The phylogenetic analyses performed on NAC sequences of 36 OGs showed congruence with the known species phylogeny (i.e. the monocot/dicot classification), even if some sequences had unexpected positions. These exceptions could be the results of specific high sequencedivergence (as suggested by their long branches in the tree) maybe due to purifying selection relaxation in the presence of redundant copies of genes.

Automated clustering by OrthoMCL with the lowest stringency level provided six clusters containing several manually obtained OGs (spanning from 4 to 10). The increase in stringency did not provide a better resolution of OGs, but tended to isolate more divergent sequences (Fig. 2). NAC sequences appear to be problematic for automatic clustering, mainly because of the large number of OGs and of repeated gene duplications inside each lineage.

The phylogenetic analysis of $O$. sativa NAC (Fang et al. 2008) was extremely consistent with the OGs' distribution obtained in this study. When several $O$. sativa NAC sequences are included in a given $\mathrm{OG}$, they also appear clustered in the phylogenetic tree obtained by Fang et al. (2008). For example, groups $1 \mathrm{a}$ and $1 \mathrm{~b}$ coincide with the two main sub-clusters of cluster I-2 (NAC1), and the six $O$. sativa NAC sequences of group $1 \mathrm{~g}$ were in a specific cluster as well as both NACs of the $1 \mathrm{~h}$ group. Some exceptions were observed involving OGs $3 \mathrm{c}, 7 \mathrm{~b}$ and $5 \mathrm{a}$. Even if NAC sequences of OGs $6 \mathrm{a}-\mathrm{c}$ and $8 \mathrm{a}-\mathrm{b}$ were resolved in five OG-specific clusters, their clusters were mixed in our phylogenetic analyses (performed with both the global sample or limited to the $6+8$ groups). This is consistent with the findings of Fang et al. (2008) limited to $O$. sativa sequences. In conclusion, no major inconsistency was observed between the outcomes of phylogeny analyses and expert definition of OGs.

NAC gene structure was found to be largely consistent with OGs based on NAC sequence similarity. A few exceptions to the three-exon structure were observed in groups 1 , 2, 3, 6 and 8. The most frequent changes are intron losses (i.e. exon mergings). One and two additional exons were observed at the beginning of the genes of OGs $7 \mathrm{a}-\mathrm{b}$ and $7 \mathrm{c}-\mathrm{d}$, respectively. Genes of groups 4,5 and 7 underwent exon/intron structure changes in the TRR, which is also very variable in its amino acid sequence. Similarity in gene structure was very common within each OG. However some differences could be observed. These differences could be explained by independent evolution of the genes (gain or loss of introns), but also they could be the results of erroneous annotations. In fact, the annotation of these genes is difficult in the very variable $\mathrm{C}$-terminal region, due to lack of similarity with other model genes. Moreover, the level of expression of these genes is often low or tissue/ condition specific (Wang et al. 2013), which reduces the representation of NAC genes in transcriptome databases.
All the sequences in each of the 40 OGs are supposed to derive from an individual ancestral gene that was present in the most recent common ancestor of monocot/dicot species. Most of the group assignations look robust. However, for some more divergent sequences, the assignation remains unreliable and further analysis involving improved annotations or additional species could modify the assignation of these sequences. The observed dicot-specific OGs (3e and $4 \mathrm{f}$ ) could be a lineage-specific evolution from another group (that could reduce the number of ancestral NAC members) or alternatively, the orthologous genes may have been lost in the monocot lineages. Even if the two dicotspecific OGs do not correspond to an ancestral sequence, at least 38 ancestral NAC sequences pre-dated the divergence of monocot/dicot lineages.

\section{NAC duplicates}

In the OG-specific phylogenies, when several sequences of a given species were included in an OG, in most of the cases these genes appeared clustered together suggesting lineage-specific amplifications. In addition, when the gene location of the sequences in their respective genome was considered, some tandem duplications were observed in $V$. vinifera (VvNAC20-21, 28-32, 51-55) (Wang et al. 2013) and in A. thaliana (ANAC003-005, 016-017, 018-019, 046-047, 048-049, 050-053, 055-056, 064-065, 067-69, 073-074, 077-078, 087-088) as well as in O. sativa (Nuruzzaman et al. 2010). Since the members of these tandem duplications are often in the same OG, it is likely that these duplications occurred after the lineage separation of the analysed species. Alternatively, some tandem duplications could be more ancient. For example, inside the phylogenetic cluster of the OG 4d, VvNAC20 + ANAC078 and VvNAC21 + ANAC077 are in a separated sub-cluster (aLRT >0.8). It is therefore likely that their tandem duplication took place before the divergence of these two dicot species (Online Resources 13). In M. acuminata, none of the detected tandem duplications could be assigned to the same OG, which indicates that their origin predates the divergence between monocots and dicots. It is worth highlighting the tandem distribution of two NAC ancestral sequences (originating OGs $1 \mathrm{~g}$ and $3 \mathrm{c}$ ) that was retained during the divergence of monocots and dicots (Fig. 5). These genes are still in tandem in all the four analysed species and they were even multiplied during the $O$. sativa and $M$. acuminata segmental duplications: VvNAC61/ANAC46/Os3g21030/Os7g48550/Os11g03370/ Os 12g03050/GSMUA_Achr3T01820/GSMUA_Ach r6T32290 (OG $1 \mathrm{~g}$ ) and VvNAC60/ANAC47/Os3g21060/ Os7g48450/Os11g03300/Os12g03040GSMUA_Achr3T0 1810/GSMUA_Achr6T32320 (OG 3c). Between the two NAC members, a RicinB gene is often found (Fig. 5). In 
Fig. 5 Evolutionary reconstruction of the fate of an ancestral locus having NAC genes of divergent OGs in tandem position. Blue and red arrowheads indicate NAC genes included in OGs $3 \mathrm{c}$ and $1 \mathrm{~g}$, respectively; green arrowheads indicate RicinB-lectin_2 genes; grey arrowheads indicate other genes

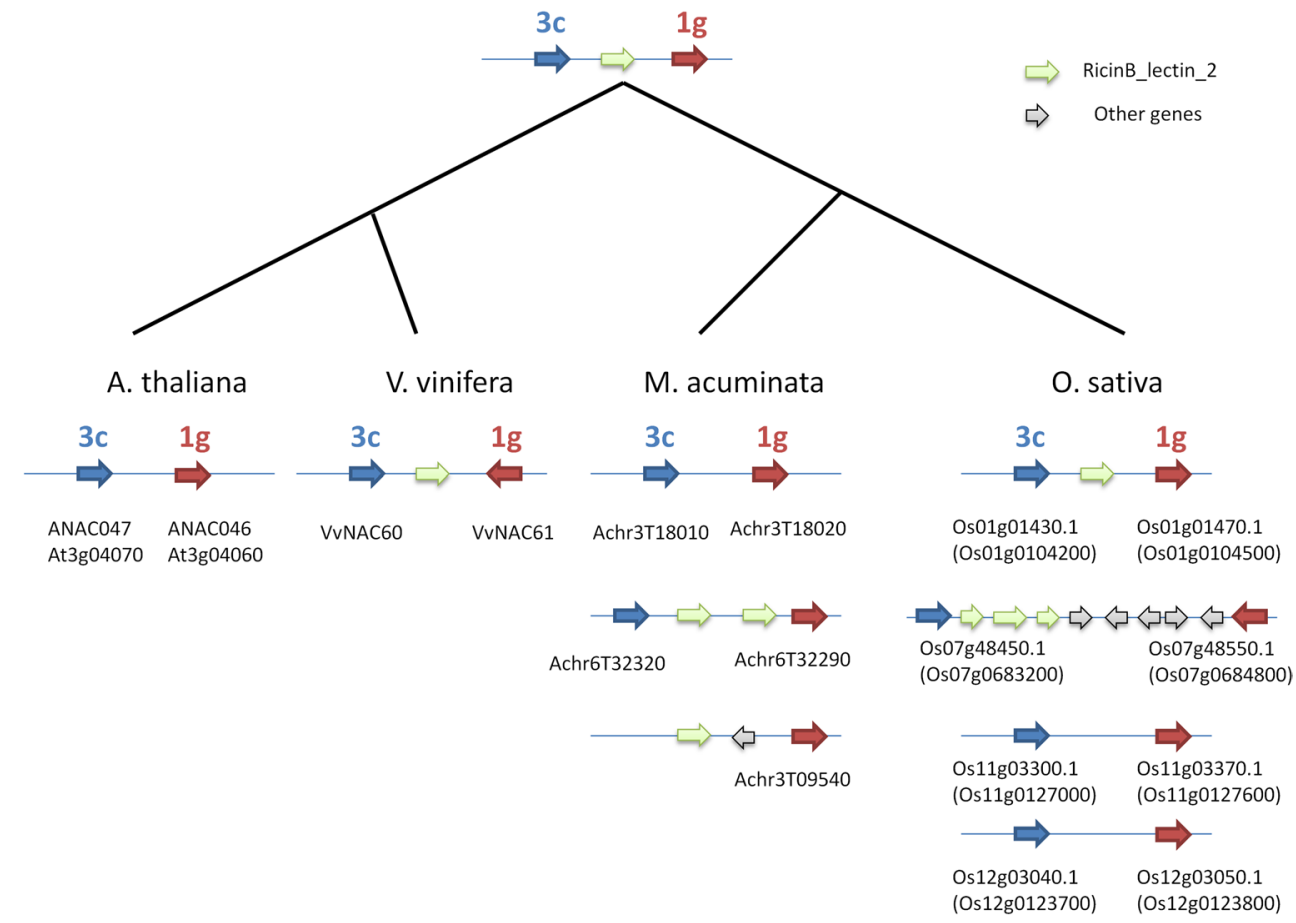

addition to the tandem position, when the $5^{\prime}-3^{\prime}$ orientation is considered, the $3 \mathrm{c}$ copies precede the $1 \mathrm{~g}$ ones in all the loci except in $V$. vinifera and $O$. sativa chromosome 7 , where the $1 \mathrm{~g}$ copies have undergone inversion (Fig. 5). Since the genes in OGs $1 \mathrm{~g}$ and $3 \mathrm{c}$ occupy very remote positions in the phylogenetic tree of all the species, one can speculate that these genes originated through more ancient tandem duplication than the monocot/dicot divergence. Alternatively, it is possible that a fortuitous rearrangement juxtaposed these genes prior to the monocot/dicot divergence. Nuruzzaman et al. (2010) already pinpointed the duplications of NAC containing regions in chr.3/chr.7 and chr.11/chr.12 in $O$. sativa. These regions coincide with known duplications in the evolution of the O. sativa genome ( $\mathrm{Yu}$ et al. 2005). By contrast, the duplication involving the ancestral regions of chr.3/chr.7 and chr.11/chr.12 was never observed. This duplication could have originated from an ancient WGD or via a segmental duplication involving a limited chromosome region. Similarly, duplication in M. acuminata was not coincident with the most recent two tetraploidization cycles (D'Hont et al. 2012) and could have originated during the older WGD suggested by D'Hont et al. (2012) or during a segmental duplication independent of any WGD.

Globally, a dramatic difference can be observed in the total number of NAC members among the four analysed taxa. Since all these species underwent to independent WGD events, the observed variability could be explained by different levels of the fractionation process (i.e. the loss of duplicated and redundant genes). If this is the main reason for the copy-number differences, similar ratios between NAC and total gene number are expected. When this ratio is calculated, $M$. acuminata showed the highest ratio $\left(4.7 \times 10^{-3}\right)$, followed by $A$. thaliana $\left(4.0 \times 10^{-3}\right)$, O. sativa $\left(3.5 \times 10^{-3}\right)$ and $V$. vinifera $\left(2.7 \times 10^{-3}\right)$. If we take in account that significant numbers of NAC genes of $O$. sativa were excluded from the comparative analysis, it appears that $V$. vinifera has a notably lower percentage of NAC genes than other species. Previous studies showed that, after WGDs, the retention of duplicated genes with regulatory functions such as transcription factors is higher than for other kinds of genes (Blanc and Wolfe 2004; Maere 2005). Consequently, the lower number of WGDs experienced by the $V$. vinifera genome could explain the observed lower genomic representation of NAC genes.

In addition to the comparison of global numbers of NAC copies, the present study allows the analysis of specific OGs. Marked differences in NAC gene number can be observed among OGs, in particular in the M. acuminata and $O$. sativa taxa (Fig. 6). These differences suggest that copy retention could vary among different OGs in a same family of transcription factors.

Potential transfer of functional gene annotation

The NAC OGs are supposed to include most of the NAC copies that have evolved from an ancestral copy existing in the most recent common ancestor of monocots and dicots. It is likely that most of these ancestral genes already had their functions, and that these functions were maintained 
Fig. 6 Hierarchical clustering of the 40 NAC OGs analysed in the four species ( $V$. vinifera, A thaliana, O. sativa and $M$. acuminata). The colour gradient from green to red indicates whether a particular group is significantly smaller or bigger based on Z-score for all genes across the four species. The figure was generated using PermutMatrix (Caraux and Pinloche 2004) with the euclidean distance and the McQuitty clustering parameters

Min $=-4.13 \quad 0.00 \quad$ Max $=4.13$

in the derived species during the independent evolution. The framework of the NAC OGs should provide a useful tool to predict the best candidates for a given function in species for which less information is available. For example, the sequence database provided in Online Resources 1 , containing the NACs of four species representative of monocots and dicots, could be used to classify any NAC sequence of a given species by a simple Blastp analysis.

In particular, NACs involved in abiotic stress-resistance or tolerance could be predicted for newly sequenced

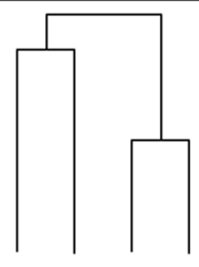

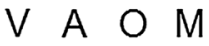

I R R U

$T$ A $Y$ Y $S$

$V$ T $S A$

I H A C

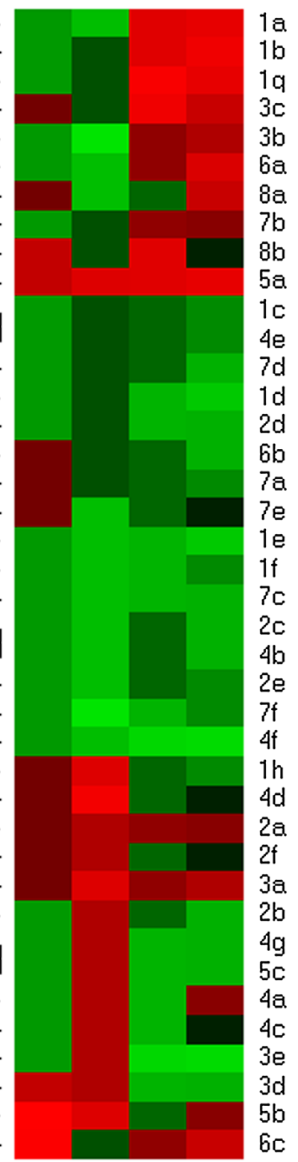

genomes such as $M$. acuminata. Based on published functions of characterized NAC genes (Table 1), one can suppose that NAC members belonging to the OGs 3,5 and 6 are mainly involved in response to biotic and abiotic stresses, even if OGs $1 \mathrm{~g}-\mathrm{h}$ appear to also play a role in this domain. Moreover many OGs do not contain any NAC genes characterized for their functions. Consequently, future results should identify an increasing number of potential genes suitable for improving the adaptability of crops to different environments and to climatic changes. 


\section{Conclusion}

The NAC gene family plays an important role in the regulation of plant development and stress-resistance/tolerance. A better understanding of the complex ancestral gene history may lead to better functional characterization of these genes. The recent sequencing of the $M$. acuminata genome has helped to elucidate the evolution of the NAC TFs in angiosperms.

Our approach to the study of NAC sequences was based on similarity analysis as inferred by a thorough and systematic examination of reciprocal Blastp results (here called expert orthologous grouping) and classical phylogenetic analysis. Phylogenetic analysis with all NAC sequences provided a global view of the reciprocal relations among NACs, but due to very divergent sequences, the resolution was insufficient to resolve some OGs. We have shown that when limiting the analysis to sequences belonging to more restricted groups, the phylogenetic resolution increases with the increase in available informative positions. Globally, phylogenetic analysis confirmed around two-thirds of the OGs based on similarity, but did not invalidate the remaining third of unresolved OGs. Combined with Blastp analysis, we were able to perform a more effective comparison between each pair of sequences with regard to the filtered multiple alignment that may not include divergent but informative sequence regions to resolve unclear OGs.

The OGs resulting from our analysis should provide a reference framework useful for functional gene annotation transfer in the NAC transcription factor family. These orthologous groups provide a curated resource for large-scale protein sequence annotation of NAC transcription factors. The established orthology relationships also provide a useful reference for NAC function studies in newly sequenced genomes such as M. acuminata and other plant species.

Acknowledgments This work was supported by the Belgian Directorate-General for Development Cooperation (DGDC) and the CGIAR Research Program on Roots, Tubers and Bananas (RTB). The authors would like to thank Vincent Johnson for his careful editing and helpful comments.

Conflict of interest The authors declare that they have no conflict of interest.

Open Access This article is distributed under the terms of the Creative Commons Attribution License which permits any use, distribution, and reproduction in any medium, provided the original author(s) and the source are credited.

\section{References}

Aida M, Ishida T, Fukaki H et al (1997) Genes involved in organ separation in arabidopsis: an analysis of the cup-shaped cotyledon mutant. Plant Cell 9:841-857. doi:10.1105/tpc.9.6.841
Anisimova M, Gascuel O (2006) Approximate likelihood-ratio test for branches: a fast, accurate, and powerful alternative. Syst Biol 55:539-552. doi:10.1080/10635150600755453

Balazadeh S, Siddiqui H, Allu AD et al (2010) A gene regulatory network controlled by the NAC transcription factor ANAC092/AtNAC2/ORE1 during salt-promoted senescence. Plant J 62:250-264. doi:10.1111/j.1365-313X.2010.04151.x

Bennett T, van den Toorn A, Sanchez-Perez GF et al (2010) SOMBRERO, BEARSKIN1, and BEARSKIN2 regulate root cap maturation in Arabidopsis. Plant Cell 22:640-654. doi:10.1105/ tpc. 109.072272

Blanc G, Wolfe KH (2004) Widespread paleopolyploidy in model plant species inferred from age distributions of duplicate genes. Plant Cell 16:1667-1678. doi:10.1105/tpc.021345

Bowers JE, Chapman BA, Rong J, Paterson AH (2003) Unravelling angiosperm genome evolution by phylogenetic analysis of chromosomal duplication events. Nature 422:433-438. doi:10.1038/ nature 01521

Bu Q, Jian H, Li CB et al (2008) Role of the Arabidopsis thaliana NAC transcription factors ANAC019 and ANAC055 in regulating jasmonic acid signaled defense responses. Cell Res 18:756-767. doi: $10.1038 /$ cr.2008.53

Caraux G, Pinloche S (2004) PermutMatrix: a graphical environment to arrange gene expression profiles in optimal linear order. Bioinformatics 21:1280-1281. doi:10.1093/bioinformatics/bti141

Carver T, Berriman M, Tivey A et al (2008) Artemis and ACT: viewing, annotating and comparing sequences stored in a relational database. Bioinformatics 24:2672-2676. doi:10.1093/ bioinformatics/btn529

Cenci A, Combes M-C, Lashermes P (2010) Comparative sequence analyses indicate that Coffea (Asterids) and Vitis (Rosids) derive from the same paleo-hexaploid ancestral genome. Mol Genet Genomics 283:493-501. doi:10.1007/s00438-010-0534-7

Cenci A, Combes M-C, Lashermes P (2013) Differences in evolution rates among eudicotyledon species observed by analysis of protein divergence. J Hered. doi:10.1093/jhered/est025

D'Hont A, Denoeud F, Aury J-M et al (2012) The banana (Musa acuminata) genome and the evolution of monocotyledonous plants. Nature 488:213-217. doi:10.1038/nature11241

Darriba D, Taboada GL, Doallo R, Posada D (2011) ProtTest 3: fast selection of best-fit models of protein evolution. Bioinformatics 27:1164-1165. doi:10.1093/bioinformatics/btr088

Delessert C, Kazan K, Wilson IW et al (2005) The transcription factor ATAF2 represses the expression of pathogenesis-related genes in Arabidopsis. Plant J Cell Mol Biol 43:745-757. doi:10.1111/j.1365-313X.2005.02488.x

Distelfeld A, Pearce SP, Avni R et al (2012) Divergent functions of orthologous NAC transcription factors in wheat and rice. Plant Mol Biol 78:515-524. doi:10.1007/s11103-012-9881-6

Droc G, Lariviere D, Guignon V, et al. (2013) The Banana Genome Hub. Database 2013:bat035. doi: 10.1093/database/bat035

Dufayard J-F, Duret L, Penel S et al (2005) Tree pattern matching in phylogenetic trees: automatic search for orthologs or paralogs in homologous gene sequence databases. Bioinformatics 21:25962603. doi:10.1093/bioinformatics/bti325

Fang Y, You J, Xie K et al (2008) Systematic sequence analysis and identification of tissue-specific or stress-responsive genes of NAC transcription factor family in rice. Mol Genet Genomics 280:547-563. doi:10.1007/s00438-008-0386-6

Gao F, Xiong A, Peng R et al (2009) OsNAC52, a rice NAC transcription factor, potentially responds to ABA and confers drought tolerance in transgenic plants. Plant Cell Tissue Organ Cult 100:255-262. doi:10.1007/s11240-009-9640-9

Guignon V, Droc G, Alaux M et al (2012) Chado controller: advanced annotation management with a community annotation system. Bioinformatics 28:1054-1056. doi:10.1093/bioinformatics/bts046 
Guindon S, Gascuel O (2003) A simple, fast, and accurate algorithm to estimate large phylogenies by maximum likelihood. Syst Biol 52:696-704

Guo Y, Gan S (2006) AtNAP, a NAC family transcription factor, has an important role in leaf senescence. Plant J 46:601-612. doi:10.1111/j.1365-313X.2006.02723.x

Guo Y, Cai Z, Gan S (2004) Transcriptome of Arabidopsis leaf senescence. Plant Cell Environ 27:521-549. doi:10.1111/j.1365-3040.2003.01158.x

He X-J, Mu R-L, Cao W-H et al (2005) AtNAC2, a transcription factor downstream of ethylene and auxin signaling pathways, is involved in salt stress response and lateral root development. Plant J Cell Mol Biol 44:903-916. doi:10.1111/j.1365-313X.2005.02575.x

Hu H, Dai M, Yao J et al (2006) Overexpressing a NAM, ATAF, and CUC (NAC) transcription factor enhances drought resistance and salt tolerance in rice. Proc Natl Acad Sci USA 103:12987-12992. doi:10.1073/pnas.0604882103

$\mathrm{Hu} \mathrm{H}$, You J, Fang Y et al (2008) Characterization of transcription factor gene SNAC2 conferring cold and salt tolerance in rice. Plant Mol Biol 67:169-181. doi:10.1007/s11103-008-9309-5

Hu R, Qi G, Kong Y et al (2010) Comprehensive analysis of NAC domain transcription factor gene family in Populus trichocarpa. BMC Plant Biol 10:145. doi:10.1186/1471-2229-10-145

Jaillon O, Aury J-M, Noel B et al (2007) The grapevine genome sequence suggests ancestral hexaploidization in major angiosperm phyla. Nature 449:463-467. doi:10.1038/nature06148

Jeong JS, Kim YS, Baek KH et al (2010) Root-specific expression of OsNAC10 improves drought tolerance and grain yield in rice under field drought conditions. Plant Physiol 153:185-197. doi:10.1104/pp.110.154773

Katoh K, Toh H (2008) Recent developments in the MAFFT multiple sequence alignment program. Brief Bioinform 9:286-298. doi:10.1093/bib/bbn013

K-i Hibara, Karim MR, Takada S et al (2006) Arabidopsis CUPSHAPED COTYLEDON3 regulates postembryonic shoot meristem and organ boundary formation. Plant Cell Online 18:29462957. doi:10.1105/tpc.106.045716

Kikuchi K, Ueguchi-Tanaka M, Yoshida KT et al (2000) Molecular analysis of the NAC gene family in rice. Mol Gen Genet MGG 262:1047-1051. doi:10.1007/PL00008647

Kim S-G, Kim S-Y, Park C-M (2007) A membrane-associated NAC transcription factor regulates salt-responsive flowering via FLOWERING LOCUS T in Arabidopsis. Planta 226:647-654. doi:10.1007/s00425-007-0513-3

Kim MJ, Park MJ, Seo PJ et al (2012) Controlled nuclear import of the transcription factor NTL6 reveals a cytoplasmic role of SnRK2.8 in the drought-stress response. Biochem J 448:353363. doi:10.1042/BJ20120244

Kubo M (2005) Transcription switches for protoxylem and metaxylem vessel formation. Genes Dev 19:1855-1860. doi:10.1101/ gad.1331305

Le DT, Nishiyama R, Watanabe Y et al (2011) Genome-wide survey and expression analysis of the plant-specific NAC transcription factor family in soybean during development and dehydration stress. DNA Res 18:263-276. doi:10.1093/dnares/dsr015

Li L, Stoeckert CJ Jr, Roos DS (2003) OrthoMCL: identification of ortholog groups for eukaryotic genomes. Genome Res 13:21782189. doi: $10.1101 /$ gr. 1224503

Lin R, Zhao W, Meng X et al (2007) Rice gene OsNAC19 encodes a novel NAC-domain transcription factor and responds to infection by Magnaporthe grisea. Plant Sci 172:120-130. doi:10.1016/j.plantsci.2006.07.019

Lyons E, Pedersen B, Kane J, Freeling M (2008) The value of nonmodel genomes and an example using SynMap within CoGe to dissect the hexaploidy that predates the rosids. Trop Plant Biol 1:181-190. doi:10.1007/s12042-008-9017-y
Maere S (2005) Modeling gene and genome duplications in eukaryotes. Proc Natl Acad Sci 102:5454-5459. doi:10.1073/p nas.0501102102

Puranik S, Sahu PP, Mandal SN, B. VS, Parida SK, Prasad M (2013) Comprehensive Genome-Wide Survey, Genomic Constitution and Expression Profiling of the NAC Transcription Factor Family in Foxtail Millet (Setaria italica L.). PLoS ONE 8: e64594. doi:10.1371/journal.pone.0064594

Mao C, Ding W, Wu Y et al (2007) Overexpression of a NAC-domain protein promotes shoot branching in rice. New Phytol 176:288298. doi:10.1111/j.1469-8137.2007.02177.x

Mitsuda N, Seki M, Shinozaki K, Ohme-Takagi M (2005) The NAC transcription factors NST1 and NST2 of Arabidopsis regulate secondary wall thickenings and are required for anther dehiscence. Plant Cell 17:2993-3006. doi:10.1105/tpc.105.036004

Mitsuda N, Iwase A, Yamamoto H et al (2007) NAC transcription factors, NST1 and NST3, are key regulators of the formation of secondary walls in woody tissues of Arabidopsis. Plant Cell 19:270280. doi:10.1105/tpc. 106.047043

Nakashima K, Tran L-SP, Van Nguyen D et al (2007) Functional analysis of a NAC-type transcription factor OsNAC6 involved in abiotic and biotic stress-responsive gene expression in rice. Plant J Cell Mol Biol 51:617-630. doi:10.1111/j.1365-313X.2007.03168.x

Nuruzzaman M, Manimekalai R, Sharoni AM et al (2010) Genomewide analysis of NAC transcription factor family in rice. Gene 465:30-44. doi:10.1016/j.gene.2010.06.008

Nuruzzaman M, Sharoni AM, Satoh K et al (2012) Comprehensive gene expression analysis of the NAC gene family under normal growth conditions, hormone treatment, and drought stress conditions in rice using near-isogenic lines (NILs) generated from crossing Aday Selection (drought tolerant) and IR64. Mol Genet Genomics 287:389-410. doi:10.1007/s00438-012-0686-8

Ochiai K, Shimizu A, Okumoto Y, Fujiwara T, Matoh T (2011) Suppression of a NAC-like transcription factor gene improves boron-toxicity tolerance in rice. Plant Physiol 156:1457-1463. doi:10.1104/pp.110.171470

Ohnishi T, Sugahara S, Yamada T et al (2005) OsNAC6, a member of the NAC gene family, is induced by various stresses in rice. Genes Genet Syst 80:135-139

Ooka H, Satoh K, Doi K et al (2003) Comprehensive analysis of NAC family genes in Oryza sativa and Arabidopsis thaliana. DNA Res 10:239-247. doi:10.1093/dnares/10.6.239

Park J, Kim Y-S, Kim S-G et al (2011) Integration of auxin and salt signals by the NAC transcription factor NTM2 during seed germination in Arabidopsis. Plant Physiol 156:537-549. doi:10.1104/ pp.111.177071

Paterson AH, Bowers JE, Bruggmann R et al (2009) The Sorghum bicolor genome and the diversification of grasses. Nature 457:551-556. doi:10.1038/nature07723

Puranik S, Sahu PP, Srivastava PS, Prasad M (2012) NAC proteins: regulation and role in stress tolerance. Trends Plant Sci 17:369381. doi:10.1016/j.tplants.2012.02.004

Ren T, Qu F, Morris TJ (2000) HRT gene function requires interaction between a NAC protein and viral capsid protein to confer resistance to turnip crinkle virus. Plant Cell 12:1917-1926

Rouard M, Guignon V, Aluome C et al (2011) GreenPhylDB v2.0: comparative and functional genomics in plants. Nucleic Acids Res 39:D1095-D1102. doi:10.1093/nar/gkq811

Saga H, Ogawa T, Kai K et al (2012) Identification and characterization of ANAC042, a transcription factor family gene involved in the regulation of camalexin biosynthesis in Arabidopsis. Mol Plant Microbe Interact 25:684-696. doi:10.1094/M PMI-09-11-0244

Seo PJ, Park CM (2011) Signaling linkage between environmental stress resistance and leaf senescence in Arabidopsis. Plant Signal Behav 6:1564-1566. doi:10.4161/psb.6.10.17003 
Shahnejat-Bushehri S, Mueller-Roeber B, Balazadeh S (2012) Arabidopsis NAC transcription factor JUNGBRUNNEN1 affects thermomemory-associated genes and enhances heat stress tolerance in primed and unprimed conditions. Plant Signal Behav 7:15181521. doi: $10.4161 / \mathrm{psb} .22092$

Shan W, J-f Kuang, Chen L et al (2012) Molecular characterization of banana NAC transcription factors and their interactions with ethylene signalling component EIL during fruit ripening. J Exp Bot 63:5171-5187. doi:10.1093/jxb/ers178

Souer E, van Houwelingen A, Kloos D et al (1996) The no apical meristem gene of Petunia is required for pattern formation in embryos and flowers and is expressed at meristem and primordia boundaries. Cell 85:159-170. doi:10.1016/S0092-8674(00)81093-4

Sun L, Zhang H, Li D et al (2013) Functions of rice NAC transcriptional factors, ONAC122 and ONAC131, in defense responses against Magnaporthe grisea. Plant Mol Biol 81:41-56. doi:10.1007/s11103-012-9981-3

Takada S, Hibara K, Ishida T, Tasaka M (2001) The CUP-SHAPED COTYLEDON1 gene of Arabidopsis regulates shoot apical meristem formation. Dev Camb Engl 128:1127-1135

Takasaki H, Maruyama K, Kidokoro S et al (2010) The abiotic stressresponsive NAC-type transcription factor OsNAC5 regulates stress-inducible genes and stress tolerance in rice. Mol Genet Genomics 284:173-183. doi:10.1007/s00438-010-0557-0

Talavera G, Castresana J (2007) Improvement of phylogenies after removing divergent and ambiguously aligned blocks from protein sequence alignments. Syst Biol 56:564-577. doi:10.1080/10635150701472164

Tang H, Bowers JE, Wang X, Paterson AH (2010) Angiosperm genome comparisons reveal early polyploidy in the monocot lineage. Proc Natl Acad Sci 107:472-477. doi:10.1073/p nas.0908007107

Tran L-SP, Nakashima K, Sakuma Y et al (2004) Isolation and functional analysis of Arabidopsis stress-inducible NAC transcription factors that bind to a drought-responsive cis-element in the early responsive to dehydration stress 1 promoter. Plant Cell 16:24812498. doi: $10.1105 /$ tpc. 104.022699

Van de Peer Y, Fawcett JA, Proost S et al (2009) The flowering world: a tale of duplications. Trends Plant Sci 14:680-688. doi:10.1016/j.tplants.2009.09.001

Wang X, Basnayake BMVS, Zhang H et al (2009) The Arabidopsis ATAF1, a NAC transcription factor, is a negative regulator of defense responses against necrotrophic fungal and bacterial pathogens. Mol Plant-Microbe Interact 22:1227-1238. doi:10.1094/ MPMI-22-10-1227

Wang N, Zheng Y, Xin H et al (2013) Comprehensive analysis of NAC domain transcription factor gene family in Vitis vinifera. Plant Cell Rep 32:61-75. doi:10.1007/s00299-012-1340-y
Willemsen V, Bauch M, Bennett T et al (2008) The NAC domain transcription factors FEZ and SOMBRERO control the orientation of cell division plane in Arabidopsis root stem cells. Dev Cell 15:913-922. doi:10.1016/j.devcel.2008.09.019

Yamaguchi M, Ohtani M, Mitsuda N et al (2010) VND-INTERACTING2, a NAC domain transcription factor, negatively regulates xylem vessel formation in Arabidopsis. Plant Cell 22:1249-1263. doi:10.1105/tpc.108.064048

Yang S-D, Seo PJ, Yoon H-K, Park C-M (2011) The Arabidopsis NAC transcription factor VNI2 integrates abscisic acid signals into leaf senescence via the COR/RD genes. Plant Cell 23:2155-2168. doi $: 10.1105 /$ tpc.111.084913

Yokotani N, Ichikawa T, Kondou Y et al (2009) Tolerance to various environmental stresses conferred by the salt-responsive rice gene ONAC063 in transgenic Arabidopsis. Planta 229:1065-1075. doi:10.1007/s00425-009-0895-5

Yoo SY, Kim Y, Kim SY et al (2007) Control of flowering time and cold response by a NAC-Domain protein in Arabidopsis. PLoS ONE 2:e642. doi:10.1371/journal.pone.0000642

Yoon H-K, Kim S-G, Kim S-Y, Park C-M (2008) Regulation of leaf senescence by NTL9-mediated osmotic stress signaling in Arabidopsis. Mol Cells 25:438-445

Yoshii M, Yamazaki M, Rakwal R et al (2010) The NAC transcription factor RIM1 of rice is a new regulator of jasmonate signaling. Plant J 61:804-815. doi:10.1111/j.1365-313X.2009.04107.x

Yoshiyama K, Conklin PA, Huefner ND, Britt AB (2009) Suppressor of gamma response 1 (SOG1) encodes a putative transcription factor governing multiple responses to DNA damage. Proc Natl Acad Sci USA 106:12843-12848. doi:10.1073/pnas.0810304106

Yu J, Wang J, Lin W et al (2005) The genomes of Oryza sativa: a history of duplications. PLoS Biol 3:e38. doi:10.1371/ journal.pbio.0030038

Yue J-X, Li J, Wang D et al (2010) Genome-wide investigation reveals high evolutionary rates in annual model plants. BMC Plant Biol 10:242. doi:10.1186/1471-2229-10-242

Zhao C, Avci U, Grant EH et al (2007) XND1, a member of the NAC domain family in Arabidopsis thaliana, negatively regulates lignocellulose synthesis and programmed cell death in xylem. Plant J 53:425-436. doi:10.1111/j.1365-313X.2007.03350.x

Zheng C, Chen B, Lu G, Han B (2009) Overexpression of a NAC transcription factor enhances rice drought and salt tolerance. Biochem Biophys Res Commun 379:985-989. doi:10.1016/j.bbrc.2008.12.163

Zhu T, Nevo E, Sun D, Peng J (2012) Phylogenetic analyses unravel the evolutionary history of NAC proteins in plants. Evol Int J Org Evol 66:1833-1848. doi:10.1111/j.1558-5646.2011.01553.x 